\title{
Perspective
}

PERSPECTIVE Actualité en histoire de l'art

1 | 2012

Art et pouvoir

\section{Art et autoreprésentation : la figure du pape entre le $\mathrm{XI}^{\mathrm{e}}$ et le $\mathrm{XIV}^{\mathrm{e}}$ siècle}

Art and self-representation: the figure of the Pope between the eleventh and fourteenth centuries

Kunst und Selbstdarstellung: Die Papstfigur zwischen dem 11. und dem

14. Jahrhundert

Arte e autorappresentazione: la figura del papa tra l'XI e il XIV secolo

Arte y autorepresentación: la figura del papa entre los siglos XI y XIV

\section{Agostino Paravicini Bagliani}

\section{OpenEdition}

Journals

Édition électronique

URL : http://journals.openedition.org/perspective/557

DOI : $10.4000 /$ perspective. 557

ISSN : 2269-7721

Éditeur

Institut national d'histoire de l'art

Édition imprimée

Date de publication : 30 juin 2012

Pagination : $95-114$

ISSN : 1777-7852

Référence électronique

Agostino Paravicini Bagliani, «Art et autoreprésentation : la figure du pape entre le xl et le xive siècle », Perspective [En ligne], 1 | 2012, mis en ligne le 30 décembre 2013, consulté le 01 octobre 2020. URL http://journals.openedition.org/perspective/557 ; DOI : https://doi.org/10.4000/perspective.557 


\title{
Art et autoreprésentation : la figure du pape entre le $\mathrm{XI}^{\mathrm{e}}$ et le $\mathrm{XIV}^{\mathrm{e}}$ siècle
}

\author{
Agostino Paravicini Bagliani
}

Dans l'histoire de la papauté médiévale, les $\mathrm{XI}^{\mathrm{e}}$-XIII ${ }^{\mathrm{e}}$ siècles occupent une place particulière. Cette période vit la réussite du projet pour lequel la papauté avait lutté depuis le début de la réforme dite grégorienne (du nom du pape Grégoire VII, 1074-1085), à savoir une triple supériorité - sur tous les évêques de la chrétienté, sur tous les souverains laïcs et sur tous les hommes - lui donnant un rôle incontesté et central au sein de l'Église universelle. C'est pour cette raison que les papes de cette période n’ont jamais utilisé le terme d' «Europe " au sens géopolitique, leur vision de la papauté s'insérant dans une perspective de la chrétienté potentiellement sans frontières (LADNER, 1983 ; PARAVICINI BAGLIANI, 2009b). Dès le début, cette stratégie ecclésiologique et cette politique de grande ampleur se sont appuyées sur une transformation complète de la figure du pape, entendue dans sa double dimension physique et institutionnelle.

L'étude de cette évolution, qui a influencé toute la suite de l'histoire de la papauté (du moins jusqu'à la fin du pouvoir temporel des papes en 1870) a constitué un itinéraire de recherche très riche au cours de ces dernières décennies, portant sur des sujets aussi différents que l'histoire des métaphores et des symboles du pouvoir, de la corporéité et de la production artistique (voir la bibliographie générale concernant l'histoire de la papauté au XIII ${ }^{\mathrm{e}}$ siècle dans PARAVICINI BAGLIANI, 2010b). La figure du pape - ou plutôt le corps du pape (PARAVICINI BAGLIANI, 1997) - a été au centre des attentions, car elle a incarné l'essentiel de la transformation de la papauté intervenue entre le haut Moyen Âge d'une part, et le bas Moyen Âge et l'époque moderne d'autre part.

\section{Le support métaphorique et symbolique}

L'étude de la symbolique du pouvoir, qui a fait l'objet d'amples recherches systématiques des années 1950 aux années 1980 (SCHRAMM, 1954-1956, 1968-1971 ; LADNER, 1941-1984), a permis de faire des progrès considérables, par exemple sur l'histoire de la chaire de saint Pierre (MACCARRONE, 1975). Une attention inédite a été accordée aux titres et aux métaphores (Vicarius Christi : MACCARRONE, 1952 ; Papa de aliquo nihil facit : KANTOROWICZ, 1961 ; "vrai empereur» : FUHRMANN, 1985 ; Soleil et lune : HAGENEDER, 2000). Les études sur le songe d'Innocent III (BERTELLI, 1989 ; GARDNER, 1989a ; VAUCHEZ, 2002), la Véronique (WOLF, 2000, 2007) ou encore l'héraldique pontificale (BOUYÉ, 2003 ; LE POGAM, 2008) ont mis toujours plus l'accent sur le lien existant entre la figure du pape et l'évolution ecclésiologique et institutionnelle, complétant ainsi, sous l'angle de la symbolique, les études sur le pouvoir

Professeur honoraire d'histoire médiévale à l'université de Lausanne, Agostino Paravicini Bagliani a consacré plusieurs ouvrages à la papauté, en particulier La Cour des papes au XIII siècle (Paris, 1995) et Le Corps du pape (Paris, 1997). Directeur scientifique de la revue Micrologus: Nature, Sciences and Medieval Societies, il étudie également l'histoire des sciences et des idées à la fin du Moyen Âge. 
pontifical qui s'étaient concentrées sur des notions appartenant plus traditionnellement aux domaines juridique et politique, telles que la "plénitude du pouvoir ", la " puissance directe " et la "puissance indirecte ", la magistrature suprême, la théocratie, la primauté de Pierre ou l'universalité (DOLCINI, 1988 ; MIETHKe, 1991 ; PARAVICINI BAGLIANI, 1996).

Que le corps du pape ait fait l'objet d'une attention particulière dès le début de la réforme dite grégorienne m'était apparu avec force en lisant un traité de Pierre Damien, l'un des principaux inspirateurs de cette réforme, dans lequel il entretenait le pape régnant, Alexandre II (1061-1073) d'un problème à première vue curieux : « Pourquoi n'arrive-t-il plus jamais au chef du Siège apostolique de vivre très longtemps, pourquoi meurt-il au contraire en un bref laps de temps ?" (PARAVICINI BAGLIANI, 1997). Les questions examinées dans ce traité intitulé De la brièveté de la vie des papes, rédigé en 1064, offraient à Pierre Damien le prétexte pour développer une rhétorique et une ritualisation de la caducité destinées à rappeler au pape régnant que son pouvoir était limité dans le temps et que la dignité qui lui avait été confiée prenait fin avec sa mort. À l'occasion de l'anniversaire du premier millénaire de la mort de saint Pierre en 1064, Pierre Damien consulta attentivement les vies des papes contenues dans le Liber pontificalis - le recueil " officiel " des biographies des papes depuis le IV siècle - et constata qu'aucun pape n'avait régné plus longtemps que Pierre, ce qui lui permit d'affirmer que la providence divine avait fixé des limites à la durée de la fonction pontificale. Après avoir proposé au pape régnant cet important élément d'autohumiliation, Pierre Damien décrivit, à la fin du traité, les rites de caducité qui étaient célébrés à la cour de l'empereur de Constantinople le jour même de son couronnement - sans doute en vue d'en proposer l'adoption par la papauté.

Dans les études menées au $\mathrm{XX}^{\mathrm{e}}$ siècle, ce traité de Pierre Damien n'a pas retenu l'attention qu'il méritait, notamment en tant que texte fondateur pour la réflexion sur la caducité du pape. Or, il s'inscrivait parfaitement, à mes yeux, dans un sillon de recherches qui avaient été inaugurées par le célèbre ouvrage de Ernst K. Kantorowicz Les Deux Corps du roi (KANTOROWICZ, 1957) et qui ont été poursuivies vingt ans plus tard par un autre historien allemand, Reinhard Elze (ELZE, 1977). Ce dernier a mis en évidence que la tension entre la dimension physique et mortelle du pape et sa continuité institutionnelle se pose différemment que pour la royauté, la papauté étant une institution non dynastique. Les propositions de Pierre Damien exigeaient cependant que la caducité du pape soit étudiée dans ses implications rituelles et métaphoriques sur la longue durée, en recourant, entre autres, aux cérémoniaux pontificaux des $\mathrm{XIII}^{\mathrm{e}}$ et $\mathrm{XV}^{\mathrm{e}}$ siècles (textes édités par Bernhard Schimmelpfennig et le père jésuite Marc Dykmans : SCHIMmelPFenNig, 1973, 2005b ; DYKMANS, 1977-1985). C'est ainsi que j'ai été amené à reconstituer, au moins jusqu'au XVI siècle, l'histoire du rituel de l'étoupe qui entra dès le XIII ${ }^{\mathrm{e}}$ siècle dans la cérémonie de consécration et du couronnement du pape, ainsi que l'histoire de la cérémonie pontificale du mercredi des cendres. Célébrée annuellement, cette cérémonie a inclus, notamment sous la papauté d'Avignon, des gestes permettant de mettre en scène la caducité du pape et la perpétuité de sa fonction.

Cette reconstitution de la ritualisation de la caducité permettait de voir comment, pendant plus de dix siècles, le corps du pape a été au centre de stratégies institutionnelles qui avaient pour fonction de résoudre symboliquement et rituellement la tension entre la caducité et la perpétuité, comme entre la fragilité corporelle et l'autorité institutionnelle. Ce discours a eu pour objectif de mettre en scène le pape comme personne mortelle mais aussi l'Église - le " corps du Christ " - comme entité éternelle ; l'humilité devenait alors connaturelle à l'exercice du pouvoir. L'étude des rituels de l'avènement du pape démontrait 
notamment l'importance symbolique de la sedes stercorata et des deux sièges que l'on croyait aux Moyen Âge être constitués de porphyre, la pierre impériale par excellence (fig. 1). Le nouveau pape devait s'asseoir sur ces deux sièges pour prendre possession du palais du Latran mais aussi pour ritualiser son accueil par les saints Pierre et Paul, les deux apôtres romains sur lesquels se fonde la primauté du pape (BOUREAU, 1988 ; PARAVICINI BAGLIANI, 1997).

L'introduction par Pierre Damien d'une rhétorique et d'une ritualisation de la caducité n'avait pourtant pas pour objectif d'affaiblir la papauté. Son traité contient justement les premières formules novatrices et audacieuses qui tendent à affirmer la triple supériorité du pape. Pierre Damien y définit en effet le pape comme étant "évêque

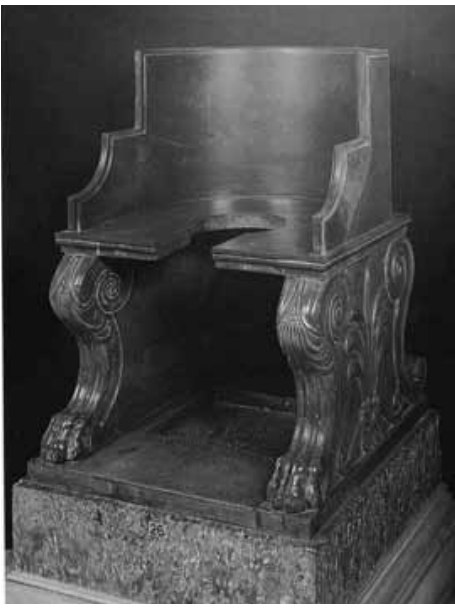
universel ", " le premier parmi les hommes ", " la tête et la cime du genre humain ". " Roi des rois et prince des empereurs ", le pape est le "seul à présider au monde entier ". Ces titres - " roi des rois ", " prince des empereurs ", " évêque universel " et " premier des hommes " - constituent un véritable programme, à la fois métaphorique et institutionnel, se fondant sur une conscience renouvelée et aiguë de la fonction pontificale. Ils concentrent les grandes aspirations du programme réformateur de la papauté de la seconde moitié du XI ${ }^{e}$ siècle (PARAVICINI BAGLIANI, 1996).

Pierre Damien a aussi été le premier à attribuer au pape le titre de Vicarius Christi jusqu'alors réservé à l'empereur, considéré comme le représentant de Dieu sur terre. Cette précision a été révélée par Michele Maccarrone dans ses travaux reconstituant l'histoire de ce titre depuis la haute antiquité chrétienne jusqu'au Moyen Âge central (MACCARRONE, 1952). Un siècle plus tard, la voie tracée par Pierre Damien s'imposa définitivement grâce à Bernard de Clairvaux. La continuité remarquable entre Pierre Damien et Bernard de Clairvaux démontre que la transformation de la figure du pape inaugurée vers le milieu du XI $\mathrm{XI}^{\mathrm{e}}$ siècle, avec un jeu inédit de métaphores, de titres et de rites, continua de se renforcer grâce à la participation des voix les plus importantes de la chrétienté. Huguccio de Pise, le plus grand canoniste de son temps, justifia vers 1190 l'attribution exclusive du titre de Vicaire du Christ en affirmant que « seul le pape peut être appelé Vicaire du Christ, si l'on considère l'autorité dont le Christ l'a investi et lui seul ". C'est encore Huguccio qui offra les arguments décisifs pour justifier la célèbre maxime selon laquelle "Rome est là où est le pape " (Ubi papa, ibi Roma). Les textes canoniques qui sous-tendent cette maxime et la définissent ont été étudiés notamment par Maccarrone et Jean Gaudemet (MACCARRONE, 1983; GAUDEMET, 1985).

L'intérêt pour le corps du pape en tant que perspective de recherche a pu se développer aussi grâce au renouveau des études sur le mécénat des papes et des cardinaux au XIII ${ }^{\mathrm{e}}$ siècle, inauguré en 1969 par Julian Gardner dans sa thèse de doctorat présentée au Courtauld Institute à Londres. Si cette étude novatrice est longtemps restée inédite, son auteur a publié par la suite de très nombreux travaux qui ont montré à quel point la production artistique promue par la cour pontificale du XIII ${ }^{\mathrm{e}}$ siècle et des premières décennies du XIV ${ }^{\mathrm{e}}$ siècle a été un instrument symbolique et figuratif au service d'une vision centrale et universelle de l'Église romaine. Ce milieu fut aussi un extraordinaire laboratoire d'idées favorisant la construction d'une identité propre aux élites romaines ainsi que la mise en place d'échanges culturels qui ont traversé la cour pontificale à un niveau international.
1. Siège de porphyre, Cité du Vatican, Musei Vaticani, Gabinetto delle Maschere. 
De nombreuses autres études, comme celle d'Alessandro Tomei (TOMEI, 2000), ont mis en évidence la place centrale de la Rome pontificale du XI ${ }^{e}$ au XIII ${ }^{e}$ siècle dans le panorama artistique européen et l'importance de la symbolique du pouvoir qui a impulsé cette production. Des synthèses ont été tentées pour expliquer ce phénomène (TOMEI, 1991 ; PACE, 2000). Les études ont surtout souligné la fonction du Latran comme lieu privilégié de la propagande et de la symbolique du pouvoir (FRUGONI, 2000 ; HERKLOTZ, 2000, 2002 ; ROMANO, 2000); la place centrale de la basilique du Vatican dans les préoccupations artistiques des papes Innocent III, Honorius III et Grégoire IX (IACOBINI, 1997) ; l'importance ecclésiologique des fresques ordonnées par Nicolas III lors des travaux de restauration de la chapelle Sancta Sanctorum (RomANo, 1992, 1995, 2000-2001, 2006 ; TOMEI, 1995) ; et la production de portraits (GANDOLFO, 2000b). De manière plus générale, la symbolique du pouvoir pontifical a été considérablement enrichie grâce à la présence d'artistes tels que Pietro di Oderisio (ClAussen, 1990), Jacopo Torriti (TOMEI, 1990), Pietro Cavallini, Giotto di Bondone (TOMEI, 2000 ; RoMANo, 2001, 2008) et Arnolfo di Cambio, architecte et sculpteur florentin sous Boniface VIII (ROMANINI, 1990, 1994, 1995a, 2001), sans oublier la très riche production artistique des miniaturistes au service du cardinal Iacopo Caetani Stefaneschi (CONDELLO, 2000 ; MADDALO, 2000).

Les études sur le mécénat des papes et des cardinaux se sont concentrées en particulier sur des figures d'artistes telles que Cavallini (TOMEI, 2000), Torriti (TOMEI, 1990), Giotto (ROMANO, 2008) ou Arnolfo di Cambio (voir, à l'occasion du sept centième anniversaire de sa mort, Arnolfo, 2005 ; Arnolfo di Cambio, 2005 ; FRANCHETTI PARDO, 2007), et sur des prélats mécènes prestigieux tels que Iacopo Stefaneschi Caetani (CONDELLO, 2000). L'axe entre Rome et Assise (ROMANO, 2001), c'est-à-dire le réseau d'influences entre la Rome pontificale et les artistes actifs dans la décoration picturale de la basilique Saint-François, notamment sous Nicolas III (1277-1280), s'est révélé être un point culminant de l'élaboration du programme idéologique de la papauté. L'importance de Nicolas III pour l'autoreprésentation artistique de la figure du pape a été confirmée par les études qui ont accompagné la restauration des fresques ordonnées par le pape Orsini dans la chapelle du Sancta Sanctorum (GARDNER, 1995), l'un des principaux lieux de la sacralité pontificale (BURKART, 2008).

L'histoire de la figure du pape sous l'angle de la corporéité a connu un élargissement considérable par des travaux portant sur le lien entre la production artistique et les sciences naturelles, en particulier l'optique (ROMANINI, 1988, 1995a, 2001). Ces études ont été impulsées par la découverte que Viterbe, où la cour pontificale séjourna fréquemment entre 1260 et 1280 (CAROCCI, 2003), a été le principal centre européen de production et de diffusion des travaux sur la perspective entre 1267 et 1277 (PARAVICINI BAGLIANI, 1991, p. 117-141) grâce à la présence d'hommes de sciences tels que le plus ancien savant polonais Witelo, le franciscain John Peckham, le dominicain Guillaume de Moerbeke et l'astronome Campanus de Novare. L'intérêt des élites curiales de cette période pour les sciences constitue aussi un point de référence obligé pour l'analyse des fresques découvertes récemment en la basilique romaine des Quatre-Saints-Couronnés (DRAGHI, 2006).

L'attention particulière des chercheurs s'est également portée sur les tombeaux et plus généralement sur l'art funéraire pontifical et cardinalice, à propos duquel nous disposons d'amples synthèses (HERKLOTZ, 1985 ; BORGOLTE, 1989; GARDNER, 1992). Le tombeau de Clément IV (1265-1268; fig. 2), qui présente le plus ancient gisant sur sol italien, a nourri la réflexion à la fois sur le lien entre l'art funéraire et le thème rhétorique du momento mori (ClAUSSEN, 1990), et sur la " renaissance de l'individu " (OLARIU, 2009). 
Mes recherches sur le thème de la caducité m'ont amené à m'intéresser à celui de la " prolongation de la vie ". Il s'avère que les textes qui ont déterminé la réécriture du mythe de l'éternelle jeunesse dans les premières décennies du XIII ${ }^{\mathrm{e}}$ siècle - grâce à l'arrivée dans l'Occident chrétien du Canon d'Avicenne et de textes tels que le "Secret des Secrets" (Williams, 2003) ainsi qu'à l'alchimie arabe - ont tous été écrits pour ou dédicacés aux élites de la société chrétienne, et notamment aux papes. Le manuscrit du plus ancien de ces traités, "Comment retarder les accidents de la vieillesse " (Paris, Bibliothèque nationale de France, ms. lat. 6978 ; PARAVICINI BAGLIANI, WILLIAMS, 1987), contient la version dédicacée au pape Innocent IV (1243-1254). De même, dans tous les traités de Roger Bacon écrits pour le deuxième pape français du XIII ${ }^{\mathrm{e}}$ siècle, Clément IV, l'auteur traite longuement de la prolongation de la vie (PARAVICINI BAgLiani, 2003a, 2009b). Il s'agit d'un phénomène culturel persistant (CRISCIANI, 2002), dont le contraste avec la rhétorique et la ritualisation de la caducité inaugurées par Pierre Damien deux siècles plus tôt n'est qu'apparent. Ces deux discours ont en effet une logique propre : le pape est mortel, et ce qui reste à sa mort, c'est l'Église, le corps du Christ (Elze, 1977) ; mais pendant son règne, l'aspiration à une santé parfaite relève

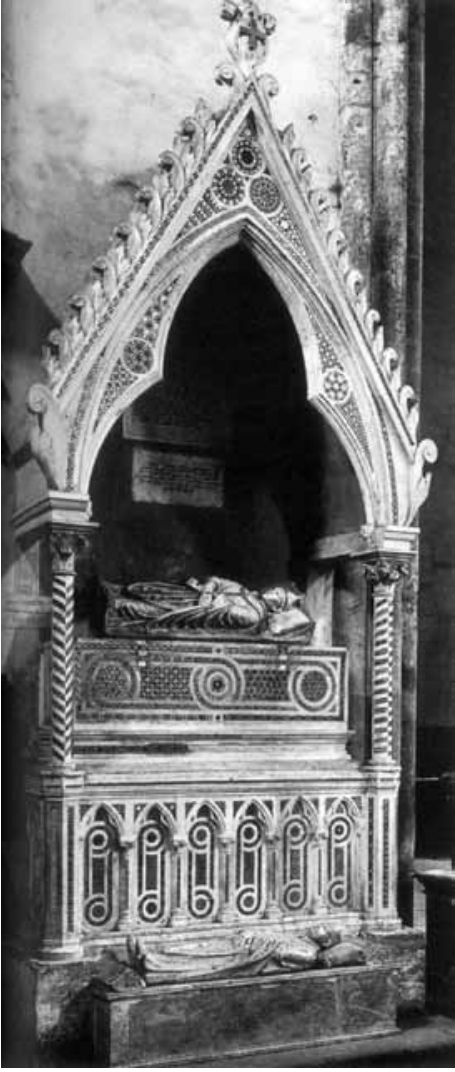

2. Tombeau de Clément IV, 1271-1274, Viterbe, Basilica di San Francesco. d'un exercice idéal du pouvoir, raison pour laquelle - comme l'a du reste dit Roger Bacon le pape, en tant que "personne publique ", mérite des soins médicaux particuliers. Nous ne sommes qu'au début d'une longue histoire qui associe le corps du souverain, le mythe de la longévité, les soins du corps du souverain et la fragilité corporelle.

\section{Autoreprésentation et art visuel sous Innocent III}

Dans son traité sur Le Mystère de l'autel sacré, c'est-à-dire sur les mystères de la messe, Lothaire de Segni, futur Innocent III, termine sa longue réflexion sur le baiser liturgique - un discours en soi inédit - en affirmant qu'il existe sept parties du corps du pape qui reçoivent des baisers liturgiques (la bouche, la poitrine, l'épaule, les mains, les bras, les pieds et les genoux). Il applique à la personne du pape une division du corps en sept parties qu'une ancienne tradition, encore bien vivante au XII siècle, avait appliqué au corps de l'Église. En décrivant les sept parties du corps du pape qui doivent être embrassées liturgiquement, Lothaire établit une identification implicite entre le corps du pape entendu dans sa dimension liturgique, et donc institutionnelle, et le corps de l'Église (PARAVICINI BAGLIANI, 1997). Lothaire inaugurait, tant sur le plan des gestes que sur celui des interprétations, un champ rituel destiné à soutenir l'autorité du pape et à fixer symboliquement les différentes hiérarchies au sein de la curie romaine et au-delà. Les sources traitant des baisers liturgiques du pape deviennent en tout cas de plus en plus nombreuses vers la fin du Moyen Âge et à la Renaissance, et constituent un domaine de l'histoire rituelle encore largement inexploité (PARAVICINI BAGLIANI, 2010a). 
$\mathrm{Au}$ fait des interrogations symboliques touchant la figure du pape, Innocent III (1198-1216) ordonna dès son avènement un programme métaphorique d'une grande ampleur au service de la fonction du pape et de son autorité, qui fut sans commune mesure avec les époques précédentes. Les objectifs de la réforme dite grégorienne restèrent inchangés, mais les moyens mis en œuvre par ce jeune pape furent riches et novateurs. Il est notamment le premier pape à réserver le titre de "Vicaire du Christ " exclusivement à la fonction pontificale (MACCARRONE, 1952) et le premier à utiliser la métaphore corporelle unissant les cardinaux au "Corps du pape ». Avec sa formule, Innocent III créait une double identification investissant la seule hiérarchie de l'Église romaine : le corps du pape, auquel les cardinaux appartiennent, fait corps avec le Christ (PARAVICINI BAGLIANI, 1997). Pour que le pape soit reconnu comme image vivante du Christ sur terre, et pour qu'il puisse exercer la "plénitude du pouvoir ", la "personne de pape " (persona pape) doit exister comme une personne en chair et en os, possédant la totalité de ses sens et pouvant être touchée, vue et "sentie" (parfumée). Dans des passages presque identiques tirés de deux sermons différents, Innocent III exprime sa conception selon laquelle la "plénitude du pouvoir " (plenitudo potestatis) et la " plénitude des sens " (plenitudo sensuum) sont liées dans la personne du pape (PARAVICINI BAGLIANI, 2009a, p. 13-20).

Innocent III a également innové sur le plan de l'autoreprésentation figurative du pape. De manière profonde et efficace, il a mis les arts visuels au service d'une conception de la papauté qui dépasse les problèmes politiques (conflits avec les empereurs, victoire de tel pape sur un rival...) - comme ce fut le cas au XII ${ }^{\mathrm{e}}$ siècle (LADNER, 1941-1984 ; HERKLOTZ, 2000) - pour se maintenir en parfaite cohérence avec l'évolution ecclésiologique en cours, si profondément engagée dans le rapprochement entre la figure du pape et celle du Christ.

\section{Le pape, le Christ et saint Pierre}

Si le premier palais résidentiel de la papauté sur la colline vaticane, édifié sous Innocent III, avait déjà suscité un regain d'intérêt au début des années 1990 (VOCI, 1992), il a plus récemment fait l'objet d'une ample monographie qui a renouvelé nos connaissances sur un aspect majeur de l'histoire pontificale (MONCIATTI, 2005). Une série d'études parues dans les années 2000 ont également inséré le palais du Vatican dans le contexte des autres résidences pontificales du XIII ${ }^{\mathrm{e}}$ siècle (LE POGAM, 2004, 2005).

Les motivations d'ordre urbanistique sont bien connues depuis la grande synthèse sur l'histoire de Rome présentée par Richard Krautheimer (KRAUTHEIMER, 2001). Le Latran, la seule demeure romaine des papes jusqu'à la construction du palais du Vatican, était décentré, mal protégé et entouré de terrains marécageux peu propices à une véritable expansion urbaine. J'ai moi-même attribué à cette situation difficile - entre autres raisons la mobilité imposée à la cour pontificale par Innocent III, qui lui permettait de passer plusieurs mois par an, entre le début de l'été et la fin de l'automne, dans une des dix villes de l'état pontifical (CAROCCI, 2003, p. 3-78). Le palais du Latran devint ainsi le "palais d'hiver " des papes, selon l'heureuse formule de Maître Grégoire (HERKLOTZ, 1985), ce clerc vraisemblablement d'origine anglaise, chapelain du cardinal piémontais Ottone da Tonengo et auteur du guide de Rome le plus intéressant des derniers siècles du Moyen Âge. Son œuvre est un témoignage saisissant de l'attention profonde et neuve que les cardinaux et les autres prélats de la cour pontificale de ces premières décennies du XIII ${ }^{\mathrm{e}}$ siècle portaient à l'esthétique, ainsi que de l'intérêt culturel qu'ils manifestaient envers les monuments antiques en relation avec la symbolique du pouvoir pontifical (NARDELLA, 1997). 
Comme l'ont rappelé Pierre-Yves Le Pogam et Sible de Blaauw, l'intérêt d'Innocent III pour le Vatican finit par modifier sensiblement le rapport entre la papauté et la ville. La résidence du Latran, malgré la marque impériale constantinienne, soulignait le fait que le pape était l'évêque de Rome, et la basilique du Latran sa cathédrale (BLAAUw, 1994 ; LE POGAM, 2004, 2005). La basilique de l'apôtre Pierre permettait en revanche de mettre en évidence l'universalité de la fonction pontificale. C'est pourquoi, en ordonnant la restauration de la grande mosaïque dans l'abside de la basilique Saint-Pierre, Innocent III fit insérer une grande inscription déclarant cette basilique mère de toutes les églises, un titre pour lequel les chanoines du Latran avaient tant combattu les siècles précédents (BLAAUW, 1994 ; WEDDIGEN, BLAAUW, KEMPERS, 2003).

La manière dont le pape Innocent III s'est fait représenter sur cette mosaïque a fait l'objet de nombreuses discussions (IACOBINI, 1997 ; PARAVICINI BAGLIANI, 2005). Dans la zone centrale de la bande inférieure furent introduites deux nouvelles figures qui apparaissent comme les chefs des deux séries d'agneaux et qui sont représentées en continuité très étroite avec le Trône et l'Agneau, en position verticale par rapport au Christ en majesté. Le pape porte la tiare, c'est-à-dire la couronne (regnum, phrygium) que Constantin aurait donnée au pape Silvestre $\mathrm{I}^{\mathrm{er}}$ (314-335) selon la célèbre Donation (fig. 3). La position des figures du pape et de l'Église romaine aux côtés du Trône avec l'Agneau, symbole du Christ, incarne l'idée selon laquelle le pape reçoit son pouvoir sur l'Église romaine directement du Christ lui-même sous forme de délégation, si on peut interpréter ainsi le geste de ses mains. Une jeune femme à la gauche du trône porte un drapeau avec les clés. Elle est aussi couronnée d'un diadème bien visible décoré de gemmes qui la désigne comme imperatrix et comme figuration de l'Église romaine.

Il s'agit de la première représentation iconographique des clés en relation avec la papauté ou, pour être plus précis, avec l’Église romaine (PARAVICINI BAGLIANI, 1998). Traditionnellement, les clés étaient un attribut iconographique de Pierre. Souvent, l'apôtre était représenté avec une ou, plus fréquemment, deux clés, qu'il tenait vers le haut ou qui pendaient de sa main par une corde. Peu après le pontificat d'Innocent III, l'iconographie des clés s'enrichit (RÉFICE, 1990). En 1229, les soldats de l'armée pontificale, qui envahirent les Pouilles, portaient l'emblème des clés et furent, de ce fait, désignés " contresignés par la clé ". Une monnaie de Charles ${ }^{\text {er }} \mathrm{d}^{\prime}$ Anjou présente deux clés tournées vers le haut et sans la corde, allusion au statut du roi de Sicile, Charles ${ }^{\mathrm{er}}$, en tant que vassal de Clément IV, c'est-à-dire de l'Église romaine. Ce même pape tient les clés comme un sceptre dans la fresque de la tour Ferrande de Pernesles-Fontaines, qui le montre en train de présenter à Charles $\mathrm{I}^{\mathrm{er}}$ la bulle avec laquelle il lui attribuait le royaume de Sicile en tant que fief pontifical (fig. 4). En 1267, dans le palais pontifical de Viterbe, apparaît la plus ancienne représentation des clés croisées (LE POGAM, 2008).

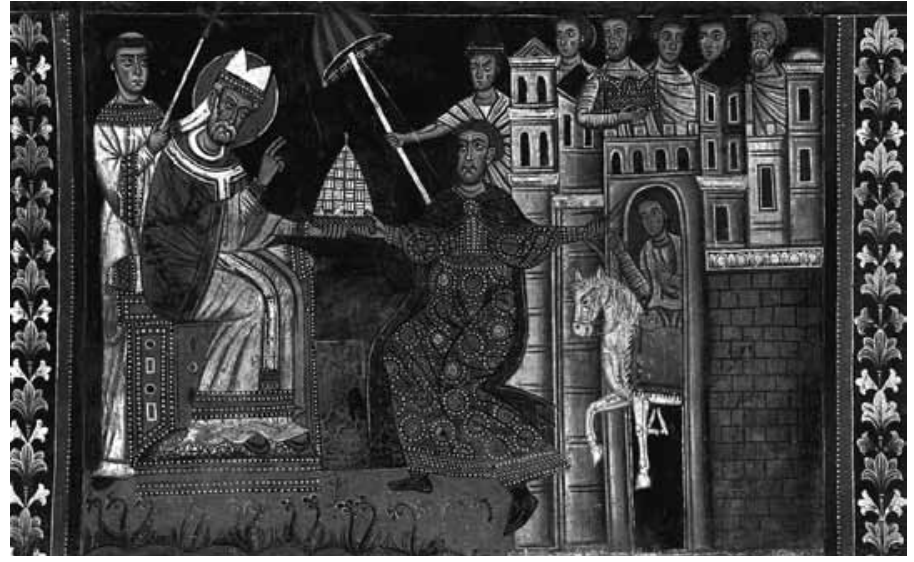

3. Donation de Constantin, XIII' siècle, Rome, Santi Quattro Coronati, cappella di San Silvestro. 


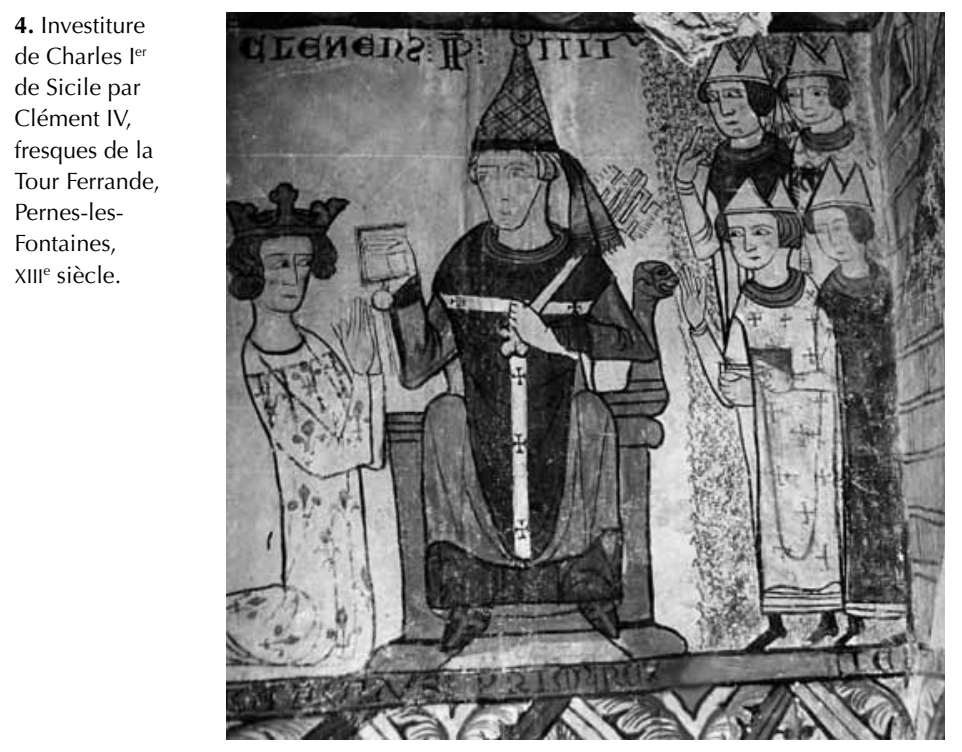

La grille de bronze située sous l'autel de la Confession au début du XIII $^{\mathrm{e}}$ siècle, en la basilique Saint-Pierre, aujourd'hui conservée au Museo Nazionale del Palazzo de Venezia, atteste également l'extraordinaire créativité d'Innocent III en termes d'autoreprésentation. Sur le côté principal de la lunette, tournée vers l'extérieur de la grille, figure au centre l'Agneau nimbé accompagné d'une inscription ("Je suis la porte [qui ouvre sur] le bercail des brebis ") inspirée d'un verset du Nouveau Testament (Jean 10:7) : "Alors Jésus dit à nouveau : 'En vérité, en vérité, je vous le dis, je suis la porte des brebis' ". Sur le cercle de l'Agneau pascal, qui met en évidence le rôle de guide et de médiateur, figure une chaire aux accoudoirs en forme de lion surmontée d'une mitre, symbole du pouvoir épiscopal. Cependant la chaire ici est pontificale puisqu'elle ressemble à celle qui se trouvait alors au centre de l'abside de la basilique vaticane (GAUTHIER, 1968). Sur le revers de la lunette, dans l'arc et l'architrave, vingt-quatre évêques sont assis sur un trône, habillés de la tunique blanche romaine et portant la mitre. Dans le médaillon central, situé derrière l'Agneau, un évêque deux fois plus grand que les autres siège sur un trône ; il n'est pas nimbé mais porte les clés de saint Pierre et, comme saint Grégoire, prête l'oreille à la colombe du Saint-Esprit. Seul à porter les clés et le pallium, il se tient au centre d'un groupe qui semble représenter l'assise des évêques rassemblés au IV concile du Latran, convoqué et présidé par Innocent III ; cette position provoque une identification inévitable entre le prince des apôtres et le pape (GAUTHIER, 1968 ; IACOBONI, 1991).

\section{Être habillé avec les couleurs du Christ}

Comme pour les autres points analysés plus haut, le discours inauguré par Innocent III se fit plus intense au cours du XIII ${ }^{\mathrm{e}}$ siècle. Vers le milieu du XIII ${ }^{\mathrm{e}}$ siècle, dans la fresque de la chapelle Saint-Sylvestre près de la basilique des Quatre-Saints-Couronnés, le pape est vêtu du manteau rouge et de la tunique blanche - nouveauté sur le plan iconographique qui coïncide chronologiquement avec les premières mentions textuelles concernant les couleurs des vêtements du pape (PARAVICINI BAGLIANI, 1997, 1998). Le blanc et le rouge renvoient à la symbolique traditionnelle des couleurs du Christ (innocence et martyr) et de l'Église romaine. Ce n'est donc pas un hasard si des textes et des images sur les vêtements blanc et rouge du pape apparaissent à une époque de très forte affirmation de la figure du pape en tant que Vicaire du Christ. Vers 1286, Guillaume Durand proposa une formulation du double vêtement du pape destinée à devenir classique : " Le pape apparaît toujours vêtu d'un manteau rouge à l'extérieur ; mais à l'intérieur, il est couvert d'un vêtement d'une blancheur éclatante ; la raison en est que la candeur signifie l'innocence et la charité ; le rouge extérieur symbolise la compassion [...] ; le pape en effet représente la personne de Celui qui pour nous teignit de rouge son vêtement ". 

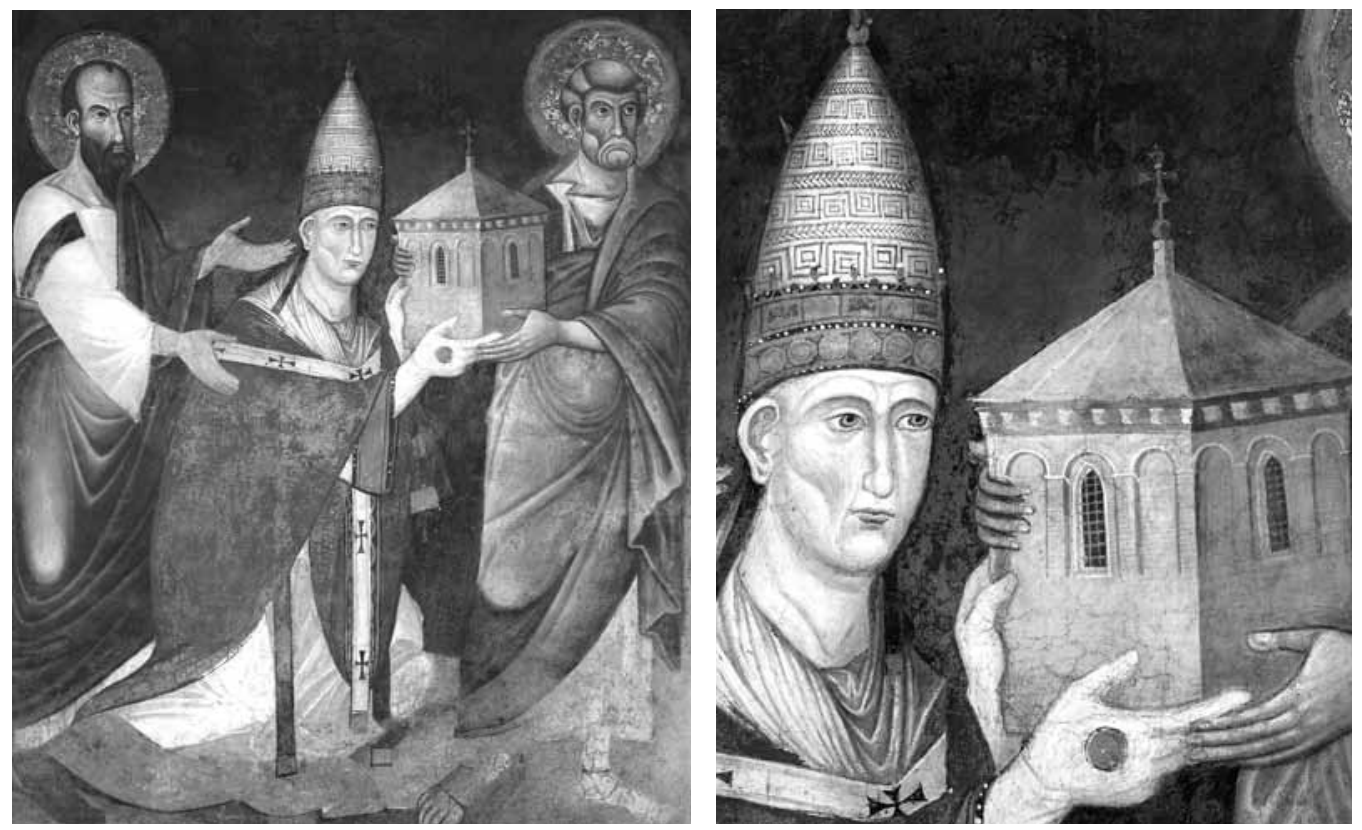

5. Nicolas III

offrant le

modèle de la

chapelle Sancta

Sanctorum au

Christ intronisé,

1278, Rome, cha-

pelle du Sancta

Sanctorum.

En termes de représentation visuelle, le programme de peintures ordonné par le pape Nicolas III dans la chapelle du Sancta Sanctorum et consacré le 4 juin, certainement avant 1280, constitue un point culminant dans la figuration du pape associé au Christ, d'une très grande efficacité artistico-ecclésiologique (GARDNER, 1995 ; ROMANO, 2000-2001). Nicolas III est représenté en effigie sur la fresque de la paroi orientale qui surplombe l'autel, ainsi que sous la voûte décorée de mosaïques qui couvre la chapelle privée du pape (fig. 5). Dans la fresque, située dans une grande lunette qui surmonte une galerie à petites colonnes, le pape, de trois quarts et à moitié agenouillé, présente un modèle de la chapelle au Christ intronisé. Saint Paul, à sa droite, lui pose la main sur l'épaule pour l'introduire en désignant son visage de la main gauche. De l'autre côté, saint Pierre l'aide à soutenir la chapelle. Les trois personnages forment un groupe compact suggérant une intimité de nature ecclésiologique, une référence claire au double fondement apostolique de la papauté romaine. Comme les saints Pierre et Paul, le pape, avec sa grande tiare, regarde vers la gauche, c'est-à-dire vers le mur où le Christ apparaît seul au centre et semble accueillir d'un geste de la main gauche les regards qui lui sont offerts. Agenouillé, Nicolas III paraît plus petit que les apôtres Pierre et Paul, mais s'il se levait, il serait bien plus grand, approchant la taille du Christ (RomANO, 2000$2001)$. Il s'agit donc d'un rapprochement profond entre le Christ et son pape, héritier de la tradition de la papauté théocratique du XIII ${ }^{\text {e }}$ siècle. Dans la chapelle du Sancta Sanctorum, cette représentation s'inscrit dans un processus visuel inédit, d'une grande puissance à la fois artistique et ecclésiologique, qui renforce l'idée du pape Vicaire du Christ, c'est-à-dire l'image du Christ sur terre (GARDNER, 1995 ; ROMANO, 2000-2001).

\section{Boniface VIII et l'apogée de l'autoreprésentation de la figure du pape au XIII siècle}

La capacité du dernier pape du XIII ${ }^{\mathrm{e}}$ siècle, Boniface VIII (1294-1303), à développer de nouvelles formes d'autoreprésentation a retenu l'attention des chercheurs, qui ont montré que 
sa puissance créatrice en termes de symbolique du pouvoir résidait en sa capacité à donner une corporéité à l'autorité qu'il incarnait et qu'il était le seul, avec son propre corps, à pouvoir représenter et mettre en scène. Boniface VIII n'a pas seulement recouru à la peinture ou à d'autres formes artistiques comme la monnaie (PARAVICINI BAGLIANI, 2007, 2009a) ; la grande nouveauté de cette forme d'autoreprésentation, qui prolonge celle de Nicolas III (ROMANO, 2006), consiste dans l'usage fréquent, régulier et systématique de la statuaire (RomANINI, 1990, 1995a, 2001). Le cardinal Caetani, futur Boniface VIII, fit donc preuve d'une grande modernité dans l'usage d'un mode de représentation, l'art funéraire, qui ne s'était imposé à la cour pontificale qu'à partir du milieu du XIII ${ }^{\mathrm{e}}$ siècle, et seulement dès le pontificat de Nicolas III pour ce qui est de la représentation sous forme de statue d'un pape régnant. Dépassant les problèmes liés à l'autoreprésentation de la papauté, ce phénomène renvoie aussi à des éléments tels que la renaissance du portrait, la cour pontificale des dernières décennies du XIII ${ }^{\mathrm{e}}$ siècle apparaissant, de ce point de vue aussi, comme un milieu particulièrement fécond (GARDNER, 2003b, 2007). Il se réfère également à la tension entre la rhétorique de la caducité, et aux thèmes du memento mori et de la glorification de soi (PARAVICINI BAGLIANI, SPIESER, WIRTH, 2007 ; OlARIU, 2009).

Pour apprécier la créativité de Boniface VIII, il a été indispensable de se pencher sur l'époque de sa première et unique légation cardinalice qui le conduisit en 1290 à Reims en compagnie de son collègue, le cardinal Gerardo Bianchi de Parme (SILANOS, 2010). Les légats devaient résoudre un conflit opposant les chanoines à l'archevêque, dont les fonctionnaires étaient accusés d'avoir séquestré des biens du chapitre. Les chanoines avaient réagi en interdisant toute célébration liturgique, jusqu'à faire taire les orgues. Le 4 décembre à l'abbaye de Saint-Cloud, les deux légats rendirent leur sentence : ils enjoignirent l'archevêque et les chanoines de faire construire deux statues d'argent, dont l'une devait représenter un cardinal évêque, Gerardo Bianchi, et l'autre un cardinal diacre, Benedetto Caetani, identifiés par leurs noms et leurs dignités, lesquels devaient figurer sur les sculptures. Outre la mitre, chaque cardinal devait porter les habits liturgiques de son rang : pour le cardinal évêque, la chasuble et les habits pontificaux, pour le cardinal diacre, la dalmatique. Chose plus singulière encore, ces deux statues devaient valoir au moins cinq cents livres tournois et être posées sur l'autel majeur, pendant la messe, lors de chaque fête solennelle de l'année liturgique. Elles ne pouvaient être ni vendues, ni même prêtées.

La question des statuettes de Reims a longtemps été considérée sous l'angle de l'idolâtrie, en raison aussi des accusations lancées contre Boniface VIII par Guillaume de Plaisians et le cardinal Pierre Colonna (COSTE, 1995). Pendant l'assemblée du Louvre, le 14 juin 1303, Plaisians dit : "Pour rendre sa mémoire très damnée perpétuelle, [Boniface] fit ériger dans des églises des statues d'argent le représentant, induisant ainsi les hommes à [l']idolâtrer " (SCHMIDT, 1986). Il ne fait aucun doute qu'en ordonnant de mettre leurs noms sur les statuettes de Reims, Gerardo Bianchi et Benedetto Caetani ont instauré une production mémorielle et personnelle. De même, le respect scrupuleux de leurs habits liturgiques, afin qu'ils soient bien distincts selon leur statut, met en évidence cette entreprise.

Des considérations d'ordre ecclésiologique ont enrichi le débat autour des statuettes de Reims, qui constituent un cas unique dans l'histoire de la papauté. Comme l'ont dit les cardinaux eux-mêmes, il s'agissait d'éviter qu'une nouvelle cessatio a divinis ne se répète ; la présence de ces statuettes à l'occasion des principales solennités liturgiques devait garantir symboliquement la continuité de l'unité de l'Église - dans ce cas entre les archevêques et les chanoines. Elles créaient un substitut interactif in absentia. Par conséquent, on peut supposer que ces statuettes servaient à perpétuer le souvenir d'un arbitrage et donc à représenter 
l'autorité judiciaire des cardinaux légats. Selon cette hypothèse, les statuettes de Reims signaleraient alors une prise de conscience des cardinaux légats de leur fonction dans la magistrature suprême de l'Église romaine (PARAVICINI BAGLIANI, 2003b).

\section{La tiare bonifacienne, sommité de l'Arche de Noé}

Une des questions les plus importantes liées à l'autoreprésentation du pouvoir pontifical, et à Boniface VIII en particulier, concerne l'évolution matérielle et symbolique de la couronne du pape - la tiare. Boniface VIII apparaît depuis longtemps comme l'introducteur d'une troisième couronne à la tiare pontificale (LADNER, 1941-1984, vol. III, 1984). Plus récemment, l'interprétation des tiares très hautes de Boniface VIII a retenu l'attention car il s'agit d'un point ayant des implications importantes sur le plan de la symbolique du pouvoir pontifical.

Dans sa description du couronnement de Boniface VIII, le cardinal Jacopo Caetani Stefaneschi avait utilisé deux mots pour définir la forme et la taille de la tiare du pape : sphère et coudée. Ce dernier mot, cubitus, renvoyait au passage de la Genèse (6:16), selon lequel la sommité de l'Arche de Noé mesurait une seule coudée. Or, le 18 novembre 1302, Boniface VIII lui-même avait utilisé cette image de l'Arche de Noé dans la bulle la plus célèbre de son pontificat, l'Unam Sanctam, pour symboliser l'unité de l'Église et réaffirmer la fonction du pape comme seul gubernator et rector : "Une fut l'Arche de Noé à l'époque du Déluge préfigurant une seule Église ; [sa sommité] se terminant en une coudée symbolise qu'elle eut Noé comme gouverneur et recteur ». Or, dans un de ses nombreux traités, Gilles de Rome, théologien sous Boniface VIII, affirme que si l'Arche de Noé représente l'Église des fidèles, la sommité de l'Arche de Noé, mesurant une coudée comme la tiare, symbolise le pape à sa tête. Ce faisant, Gilles de Rome transférait au pape ce que plus d'un siècle plus tôt le théologien Hugues de Saint-Victor, dans son célèbre traité sur l'Arche de Noé, avait attribué au Christ (PARAVICINI BAGLIANI, 2009a). Pour Boniface VIII, porter une tiare si haute signifiait donc positionner le pape à la sommité de l'Église. Cette réinterprétation de la tiare comme symbole de la plus haute hiérarchie dans l'Église constitue une transformation en profondeur des interprétations symboliques plus anciennes qu'Innocent III avait résumées en disant que la tiare est "le symbole du pouvoir impérial " du pape (signum imperii), et la mitre le symbole de sa fonction sacerdotale (signum sacerdotii).

La plus ancienne représentation du couronnement d'un pape, sur les gradins devant la basilique Saint-Pierre du Vatican, figure dans un des manuscrits du traité contenant la description du couronnement de Boniface VIII par le cardinal Stefaneschi (PARAVICINI BAGLIANI, 1998 ; fig. 6) ; ce texte a été scrupuleusement suivi par le miniaturiste. Devant le portique de la basilique vaticane (dont certains détails architectoniques se retrouvent, au moins en partie, dans les reconstructions de la façade médiévale : MADDALO, 1997), Benedetto Caetani, élu pape Boniface VIII, reçoit la tiare à double couronne des mains de Matteo Rosso Orsini, le plus ancien des cardinaux diacres, comme le voulait la tradition. Le nouveau pape porte le manteau rouge et le pallium blanc en forme de croix, la dalmatique violette, les pantoufles blanches et

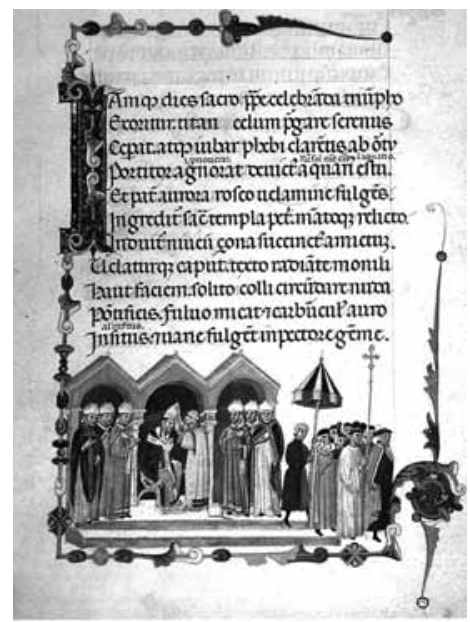




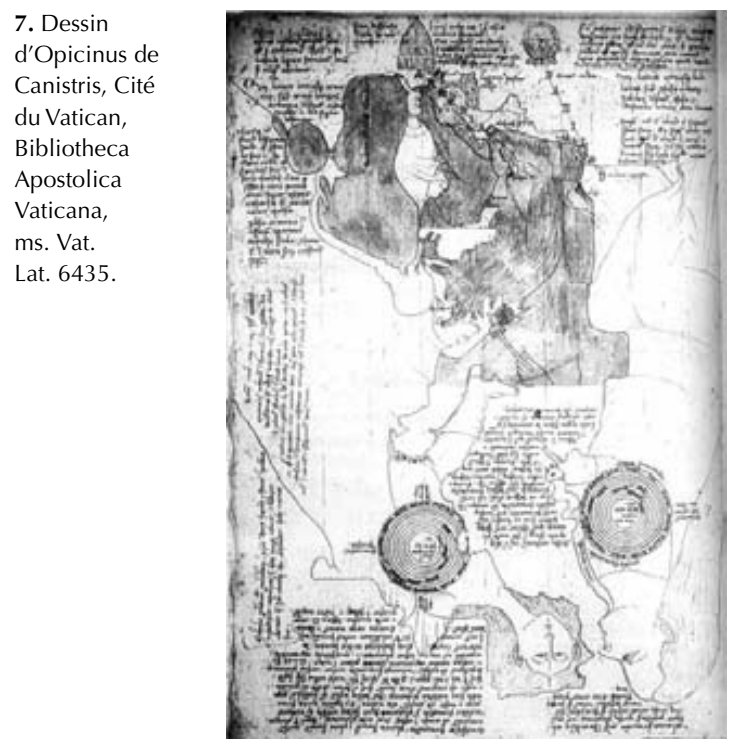

des gants blancs. Aux pieds du cardinal diacre figure la mitre que le cardinal avait enlevée de la tête du pape pour y poser la couronne, appelée regnum. Certains historiens de l'art identifient le prédécesseur de Boniface VIII, Pierre de Morrone-Célestin V, comme le personnage barbu et au-dessus duquel un acolyte porte le dais, mais cette interprétation continue à faire l'objet de débats. S'agit-il de Célestin V ou bien de la figure du prélat qui doit ouvrir la procession pour porter le nouveau pape couronné du Vatican au Latran? Comme l'a soulevé Valentino Pace, la question reste ouverte (PACE, 2000).

J'ai eu l'occasion de démontrer récemment que l'interprétation de la tiare comme symbole de la sommité de l'Arche de Noé a connu un certain succès même à Avignon. Dans deux de ses magnifiques dessins, Opicinus de Canistris dessina en effet la tiare en l'identifiant explicitement à l'Arche (fig. 7). Dans deux autres, il accompagna la figure du pape par une inscription qui l'assimile au " corps apostolique de l'Église ». La figure du pape est analogue à celle du "fils de l'homme ", c'est-à-dire, le Christ (PARAVICINI BAGLIANI, 2009a, p. 227-235). En identifiant le pape au Christ, Opicinus de Canistris - dont l'œuvre (contenue dans le ms. Vat. Lat. 6435 de la Bibliothèque apostolique vaticane) a fait récemment l'objet d'une édition et d'une traduction française (LAHARIE, 2008) - représente le pape comme l'incarnation de l'Église.

\section{Boniface VIII bénissant comme le Christ et tenant les clés comme saint Pierre}

Les statues de Boniface VIII ont suscité l'intérêt des historiens et des historiens de l'art, notamment à l'occasion des célébrations commémorant le sept centième anniversaire de la mort d'Arnolfo di Cambio, le plus grand sculpteur italien autour de 1300 (Arnolfo, 2005 ; Arnolfo di Cambio, 2005). Le gisant et le buste de la chapelle funéraire de Boniface VIII dans la basilique vaticane ainsi que la statue du pape réalisée pour la façade de la cathédrale de Florence, offrent des traits dont ne peut pas dire qu'il s'agisse de ceux du pape (fig. 8-9). Si la thèse de Leonard Boyle est juste, Arnolfo di Cambio (ou un membre de son atelier) serait aussi l'auteur du bas-relief présentant un pape nimbé, dont les traits sont très ressemblants aux autres statues déjà citées, et qui serait un cryptoportrait de Boniface VIII (BoYLE, 1978).

À la suite des analyses systématiques de Gerhart B. Ladner, c'est surtout le gisant qui a retenu l'attention (LADNER, 1941-1984). Pour Michele Maccarrone, cette sculpture, placée dans une chapelle que le pape voulut une "représentation visible et concrète de son pontificat ", donne l'image d'un corps en " fonction de la papauté " (MACCARRONE, 1991, vol. II, p. 1206-1247). Angiola Maria Romanini a été frappée par la très haute qualité du travail ainsi que par la puissance, très moderne, de ce que l'on peut qualifier de " portrait authentiquement psychologique ", exceptionnel même par rapport au reste de l'œuvre d'Arnolfo di Cambio (ROMANINI, 1995a). Le visage du gisant porte les traits physionomiques de Boniface VIII, comme en témoignent les comparaisons avec d'autres statues; son " calme classique est sérénité qui vainc la mort ", une image qui fond l'éternel dans le présent (ROMANINI, 1990). 
Le buste de Boniface VIII, œuvre d'Arnolfo di Cambio, est la première statue d'un pape qui bénit de la main droite et porte les clés de saint Pierre de la main gauche. À ce propos, Romanini a démontré que le poing gauche serré autour des deux clés est semblable à celui de la statue en bronze de l'apôtre Pierre que les fidèles vénéraient dans la basilique vaticane, une analogie qui confirme la possibilité d'attribuer la statue en bronze de saint Pierre au même artiste (ROMAnINI, 1995a, 2001). La monnaie que Boniface VIII fit frapper en 1301, à Pontde-Sorgues, dans le comtat Venaissin, présente sur le côté droit le buste du pape - première dans l'histoire des monnaies pontificales - qui porte une tiare très haute, avec les clés dans la main droite (TRAVAINI, 2004). Le buste vatican met en scène sur le plan tridimensionnel une ecclésiologie parmi les plus audacieuses qui aient été tentées au Moyen Âge, ce qui en fait un document visuel d'une extrême intensité symbolique.

Nancy Rash (RASH, 1987) a insisté sur le fait que le buste d'Arnolfo di Cambio représente Boniface VIII grandeur nature et mesure plus d'un mètre de haut. Les bordures ornées du pluvial révèlent ses mains et accentuent la largeur de la base, attirant également l'attention sur le visage, dont la physionomie semble en accord avec celle que l'on voit sur son gisant et sur certaines des autres statues le représentant. De manière encore plus forte que dans les statuettes de Reims, le buste d'Arnolfo di Cambio crée une véritable dynamique entre un corps puissant et un portrait volontairement reconnaissable d'une part, et une symbolique d'une forte intensité ecclésiologique d'autre part. À cela s'ajoute le fait que, selon une considération récente de Serena Romano (RomANo, 2000-2001, 2006), la statue en bronze de saint Pierre présente une nouveauté : le geste de la main qui bénit. Romano affirme qu'il existe deux œuvres d'Arnolfo di Cambio - la statue en bronze et le buste de Boniface - qui s'emparent de ce geste, lequel, dans la tradition iconographique, avait appartenu exclusivement au Christ. Elle ajoute : "Et parmi ces deux œuvres, celle qui plus ouvertement révèle l'ascendance christologique du modèle est justement le
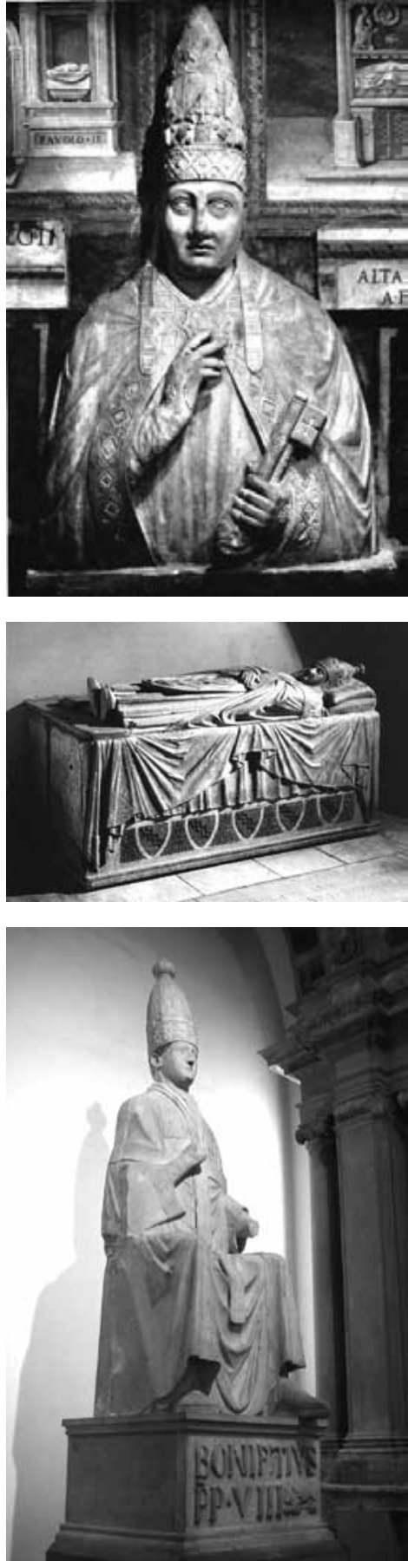
portrait bonifacien ", qui pourrait être mis en relation avec le très célèbre portrait du Christ pré-iconoclaste du monastère Sainte-Catherine sur le Mont Sinaï.

Tout cela nous rappelle l'extraordinaire richesse du tombeau de Boniface VIII, œuvre d'Arnolfo di Cambio. L'ensemble des éléments - l'architecture, le gisant, le buste et la mosaïque de Jacopo Torriti - participe à affirmer la vision de la papauté telle que la concevait
8. Arnolfo

di Cambio, Boniface VIII, 1301, Rome, San Pietro in Vaticano :

a. buste ;

b. gisant.

9. Arnolfo di Cambio, Boniface VIII, 1298, Florence, museo dell'Opera del Duomo. 
le dernier pape du XIII ${ }^{\mathrm{e}}$ siècle, comme l'ont montré les recherches de ces trois dernières décennies en histoire (MACCARRONE, 1983 ; PARAVICINI BAGLIANI, 2009a) et en histoire de l'art (ROMANINI, 1990, 1995a ; TOMEI, 2000).

\section{Le pape bénissant et maudissant}

Une autre grande question concernant Boniface VIII et son autoreprésentation a donné lieu à de multiples discussions parmi les chercheurs (MADDALO, 1998-1999 ; FRUGONI, 2000 ; GANDOLFO, 2000a ; PARAVICINI BAGLIANI, 2005, 2009a). Elles portent sur les raisons qui ont poussé le pape à faire édifier une loggia adossée au palais du Latran, et de se faire représenter au sein de cette structure dans une fresque visible sur le premier pilier droit de la nef centrale de la basilique Saint-Jean-de-Latran (fig. 10). Le pape, qui porte la tiare et bénit la foule de la main droite, est accompagné à sa droite par un cardinal et à sa gauche par un clerc qui tient un phylactère avec une inscription : Bonifacius. ep(iscopu)s. seruus seruoru(m) dei ad perpetuam rei memoria $(m)$.

Il s'agit de l'unique fragment conservé d'un cycle de fresques qui était à l'origine de dimensions considérables. L'image centrale du cycle nous a été transmise grâce à une copie réalisée vers la fin du XVI ${ }^{\mathrm{e}}$ siècle par le chanoine de Saint-Pierre au Vatican, Giacomo Grimaldi, maintenant conservée dans un manuscrit de la bibliothèque ambrosienne de Milan (ms. F. inf. 227, fol. 3r ). Les deux autres scènes étaient consacrées à la fondation de la basilique du Latran et au baptême de Constantin. Le pape est vêtu de son manteau rouge et porte la tiare à deux diadèmes. La position de la main droite semble indiquer qu'il est en train de bénir, soit pour concéder une indulgence, soit pour donner l'absolution ou pour prêcher. À sa droite et à sa gauche se tiennent deux groupes d'ecclésiastiques, dont la plupart portent des vêtements liturgiques blancs. Au fond cinq sergents du pape tiennent des hallebardes.

L'une des plus anciennes interprétations de cette fresque, et aussi la plus connue, affirme que la scène représentée est celle de la promulgation du premier jubilé chrétien en 1300 par Boniface VIII. Selon une autre interprétation, avancée par Silvia Maddalo,
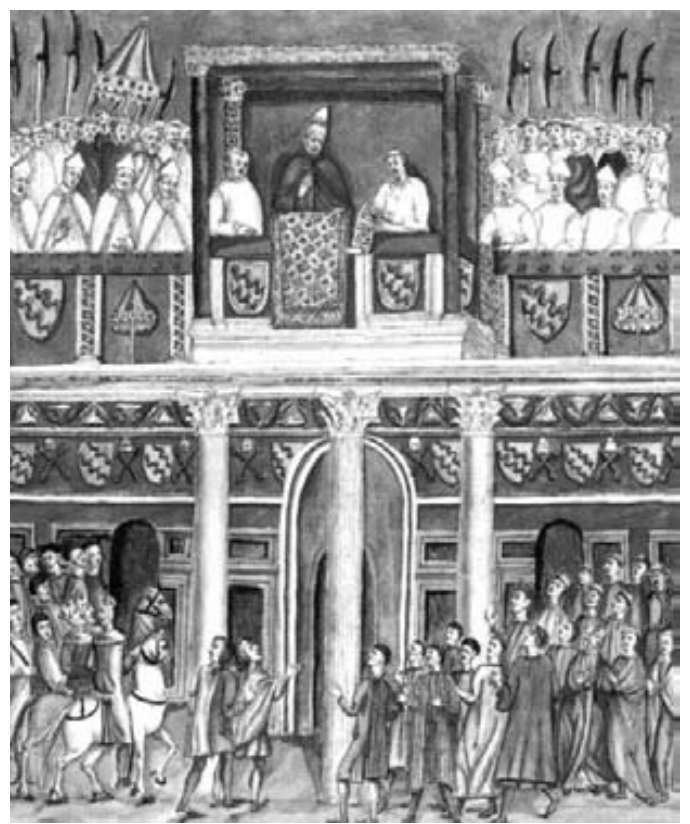

Boniface VIII serait en train de bénir au terme de la prise de possession du Latran (MADDAlo, 1997, 1998-1999). Chiara Frugoni a mis récemment en évidence le fait que les mots du phylactère ( $a d$ perpetuam rei memoriam) sont identiques à ceux qui se trouvent au début de la bulle par laquelle Boniface VIII, le Jeudi saint 1300, a renouvelé l'excommunication des Colonna et les a privés des bénéfices du jubilé (FRUGONI, 2000). Pour ma part, j'ai fait remarquer que les registres de lettres de Boniface VIII contiennent un nombre très élevé de lettres précédées par cette formule et que, dans la majorité des cas, leur date coïncide avec l'une des trois fêtes - Jeudi saint, Ascension, 18 novembre (fête de la dédicace des basiliques SaintPierre et Saint-Paul) - au cours desquelles 
le pape, accompagné de la curie romaine, célébrait le rituel destiné à excommunier les rebelles de l'Église (PARAVICINI BAGLIANI, 2005). La célébration régulière de ce rite par Boniface VIII, même à des dates non prévues, incite à relire les cérémoniaux pontificaux contenant la description de ce rite (depuis l'ordo XIII, rédigé pour le pape Grégoire X, 1271-1276 : DYKMANS, 1977-1985), ce qui permet de constater au moins dix points communs avec le dessin de Grimaldi : le pape se rend à la cérémonie, précédé par une croix ; il porte le manteau rouge ; tous les cardinaux et les prélats de la curie doivent porter des vêtements blancs; le cardinal diacre, à la gauche du pape, expose, en langue vulgaire, la bulle du procès ; le chapelain à la droite du pape lit la bulle d'excommunication, des chandelles sont allumées ; les prélats regardent vers le peuple ; à la fin de la cérémonie, le pape se tourne vers le peuple et donne l'absolution ou l'indulgence ; le pape part, précédé par la croix. Ces coïncidences jettent une lumière nouvelle sur le sens de la fresque et sur la loggia elle-même, que Boniface VIII semble avoir fait édifier, peut-être au cours de l'an 1300, en tant que loggia de justice, en vue de la célébration des procès généraux d'excommunication des rebelles de l'Église. Sur la fresque, à la gauche du pape, on n'avait jamais noté que derrière les quatre prélats avec mitre, se tient un clerc qui porte une torche allumée, que le pape devait jeter dans la foule au cours du rituel.

Comme l'a rappelé Richard Krautheimer (KRAUTHEIMER, 2001), les papes des dernières décennies du XIII ${ }^{\mathrm{e}}$ siècle - avant tout Nicolas III et Boniface VIII - ont voulu faire de la Rome pontificale la capitale artistique de la chrétienté, en confiant la réalisation d'œuvres à des artistes tels que Pietro Cavallini, Iacopo Torriti et Arnolfo di Cambio. La mort de Boniface VIII en 1303, le pontificat relativement éphémère de Benoît XI (1303-1304) et surtout la décision de Clément V et de ses successeurs d'établir la papauté à Avignon, du moins temporairement, ont porté un coup d'arrêt radical aux grandes entreprises artistiques et d'autoreprésentation de la papauté romaine. Les papes d'Avignon, de 1305 à 1377, ne disposaient plus des grands lieux symboliques de la Rome pontificale, surtout les basiliques du Latran et de Saint-Pierre. L'une des chapelles les plus importantes du palais des papes à Avignon a cependant été dédiée à saint Jean (pour les célèbres fresques de Matteo Giovannetti, voir CASTELNUOvo, 1996). Une relecture attentive des fresques de la bibliothèque privée de Clément VI par Étienne Anheim (ANHEIM, 2008) a montré récemment que la symbolique liée à la personne de l'apôtre Pierre (en tant que pêcheur d'hommes) constitue la trame de fond de ces ouvres célèbres, qui ont souvent été lues plutôt comme un témoignage exceptionnel de l'intérêt nouveau que le pape et les élites de la cour pontificale d'Avignon portaient à la nature. La tiare elle-même atteint une forme définitive, de trois couronnes identiques, sous le pape Clément VI (1342-1352 ; LADNER, 1941-1984, vol. III), ce qui montre à quel point la papauté d'Avignon s'inscrit dans la continuité pour ce qui est de l'autoreprésentation et de la symbolique du pouvoir, y compris dans le domaine des portraits (ANHEIM, 2007). En ce qui concerne la ritualisation, on constate aussi une grande continuité et d'inévitables adaptations spatiales, notamment lors des cérémonies de couronnement (SCHIMMELPFENNIG, 1990).

Les divergences sont également nombreuses. La Rose d'or était depuis le $\mathrm{XI}^{\mathrm{e}}$ siècle le cadeau le plus prestigieux que pouvait offrir un pape. Le quatrième dimanche du Carême, les papes conféraient la Rose d'or au préfet de la ville ou à un souverain, dans le cadre d'un rituel relativement complexe dont la structure n'a pas vraiment varié au cours du bas Moyen Âge et de la Renaissance. Le pape, coiffé d'une mitre de fête, allait chanter à l'église 
11. Rose d'or, 1330, Avignon, Paris, Musée de Cluny-musée national du Moyen Âge.

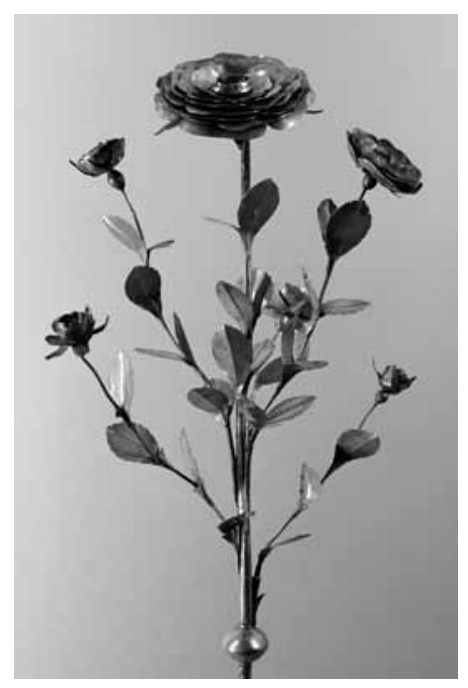

Sainte-Croix-de-Jérusalem " où s'il est ailleurs, là où il lui plaira ". Ce jour-là, le pape portait en main une Rose d'or, admirable travail d'orfèvrerie. À Avignon, les papes ont continué à donner chaque année la Rose d'or à une personnalité de choix, mais la nature de ces destinataires s'est profondément modifiée. Une étude de ces derniers révèle en effet que ce don prestigieux a servi à l'édification de nouveaux réseaux de protection (PARAVICINI BAGLIANI, 2009d). La Rose d'or la plus ancienne qui soit parvenue jusqu'à nous (1330, Paris, Musée de Cluny ; fig. 11) a été longtemps considérée comme étant celle que le pape Clément V (13051314) aurait offerte à l'évêque de Bâle. Il s'agit en fait de la Rose d'or que Jean XXII (1318-1334) donna au comte de Neuchâtel de la ligne des seigneurs de Nidau dans la Suisse actuelle (comiti de Nienborc; OTAVSKY, 1986).

Les recherches récentes sur l'autoreprésentation et la symbolique du pouvoir pontifical des papes d'Avignon démontrent à quel point l'institution pontificale eut recours à des opérations métaphoriques et iconographiques pour affirmer, dans la capitale rhodanienne, que le pape demeurait évêque de Rome et Vicaire du Christ, et pour transférer à Avignon une part de la majesté romaine. En général, les recherches dont il a été fait état plus haut démontrent aussi que le programme métaphorique et iconographique de la papauté d'Avignon prolonge l'extraordinaire entreprise métaphorique et visuelle réalisée par les papes du XIII ${ }^{\text {e }}$ siècle. Cette opération avait notamment pour but d'assurer les grands objectifs du programme réformateur de la papauté tels qu'ils avaient été élaborés dès le milieu du XI ${ }^{\mathrm{e}}$ siècle et clairement esquissés dans le traité de Pierre Damien, à savoir la centralité de l'Église romaine et sa triple supériorité, sur tous les évêques, sur tous les souverains laïques et sur tous les hommes. 


\section{Bibliographie}

- Anheim, 2007 : Étienne ANHeim, " I ritratti dei papi tra Roma e Avignone ", dans FRANCHETTI PARDO, 2007, p. 231-238. - ANheim, 2008 : Étienne ANHeim, " La Chambre du Cerf : image, savoir et nature à Avignon au milieu du XIV ${ }^{\text {e }}$ siècle ", dans Micrologus, 16, 2008, p. 57-124. - Arnolfo, 2005: Arnolfo: alle origini del Rinascimento fiorentino, Enrica Neri Lusanna éd., (cat. expo., Florence, Museo dell'Opera di Santa Maria del Fiore, 2005-2006), Florence, 2005.

- Arnolfo di Cambio, 2005 : Arnolfo di Cambio: una rinascita nell'Umbria medievale, Vittoria Garibaldi, Bruno Toscano éd., (cat. expo., Pérouse, Galleria Nazionale dell'Umbria/Orvieto, chiesa di Sant'Agostino, 2005-2006), Milan, 2005.

- Bertelli, 1989 : Carlo Bertelli, "Römische Träume ", dans Agostino Paravicini Bagliani, Giorgio Stabile éd. Träume im Mittelalter: ikonologische Studien, Stuttgart/Zurich, 1989, p. 91-112.

- BlaAuw, 1994 : Sible de Blaauw, Cultus et decor: liturgia e architettura nella Roma tardoantica e medievale, Cité du Vatican, 1994.

- Borgolte, 1989 : Michael Borgolte, Petrusnachfolge und Kaiserimitation: Die Grablegen der Päpste, ihre Genese und Traditionsbildung, Göttingen, 1989.

- Boureau, 1988 : Alain Boureau, La Papesse Jeanne, Paris, 1988.

- Bouyé, 2003 : Édouard Bouyé, "Les armoiries pontificales à la fin du XIII ${ }^{\mathrm{e}}$ siècle. Construction d'une campagne de communication ", dans Médiévales, 44, 2003, p. 173-198.

- Boyle, 1964 : Leonard E. Boyle, " An Ambry of 1299 at San Clemente, Rome ", dans Mediaeval Studies, 26, 1964, p. 329-350.

- BURKART, 2008 : Lucas Burkart, Das Blut der Märtyrer: Schatz und Schatzbildung in Gesellschaften des Mittelalters, Cologne, 2008.

- CARocci, 2003 : Sandro Carocci, Itineranza pontificia: la mobilità della curia papale nel Lazio (secoli XII-XIII), (Nuovi studi storici, 61), Rome, 2003.

- CARRÉ, 1993 : Yannick Carré, Le Baiser sur la bouche au Moyen Âge : rites, symboles, mentalités, à travers les textes et les images, $X I^{e}-X V^{e}$ siècles, Paris, 1993.

- CAstelnuovo, 1996 : Enrico Castelnuovo, Un peintre italien à la cour d'Avignon: Matteo Giovannetti et la peinture en Provence au XIVe siècle, Paris, 1996.
- Claussen, 1990 : Peter Cornelius Claussen, "Pietro di Oderisio und die Neuformulierung des italienischen Grabmals zwischen Opus Romanum und Opus Francigenum ", dans Jörg Garms, Angiola Maria Romanini éd., Skulptur und Grabmal des Spätmittelalters in Rom und Italien, (colloque, Rome, 1985), Vienne, 1990, p. 173-200.

- Condello, 2000 : Emma Condello, " Libri e committenza nella Roma del primo Giubileo: i codici Stefaneschi e dintorni ", dans Bonifacio VIII e il suo tempo: anno 1300 il primo Giubileo, Marina Righetti Tosti-Croce éd., (cat. expo., Rome, Palazzo di Venezia, 2000), Milan, 2000, p. 103-106.

- Coste, 1995 : Jean Coste éd., Boniface VIII en procès : articles d'accusation et dépositions des témoins (1303-1311), Rome, 1995.

- CRISCIANI, 2002 : Chiara Crisciani, Il papa e l'alchimia: Felice V, Guglielmo Fabri e l'elixir, Rome, 2002.

- DolCini, 1988 : Carlo Dolcini, Crisi di poteri e politologia in crisi: da Sinibaldo Fieschi a Guglielmo d'Ockham, Bologne, 1988. - DRAGHI, 2006 : Andreina Draghi, Gli affreschi dell'Aula Gotica del monastero dei Santi Quattro Coronati: una storia ritrovata, Milan, 2006.

- DYKMANS, 1977-1985 : Marc Dykmans éd., Le Cérémonial papal de la fin du Moyen Âge à la Renaissance, 4 vol., Bruxelles/ Rome, 1977-1985.

- Elze, 1977 : Reinhard Elze, "Sic transit gloria mundi: la morte del papa nel Medioevo ", dans Annali dell'Istituto storico italo-germanico in Trento. Jahrbuch des italienisch-deutschen historischen Instituts in Trient, 3, 1977, p. 23-41.

- FRANChetTI PARDo, 2007 : Vittorio Franchetti Pardo éd., Arnolfo di Cambio e la sua epoca: costruire, scolpire, dipingere, decorare, (colloque, Florence, 2006), Rome, 2007.

- FRUgoni, 2000 : Chiara Frugoni, Due papi per un Giubileo: Celestino V, Bonifacio VIII e il primo Anno Santo, Milan, 2000. - FuHRMANN, 1985 : Horst Fuhrmann, "'Der wahre Kaiser ist der Papst'. Von der irdischen Gewalt im Mittelalter", dans Hans Bungert éd., Das antike Rom in Europa: Die Kaiserzeit und ihre Nachwirkungen, Ratisbonne, 1985 p. 99-121.

- GANDOLFO, 1999 : Francesco Gandolfo, «Bonifacio VIII, il Giubileo del 1300 e la
Loggia delle Benedizioni al Laterano ", dans Romei e Giubilei: il pellegrinaggio medievale a San Pietro (350-1350), Mario d'Onofrio éd., (cat. expo., Rome, Palazzo Venezia, 2000), Milan, 1999, p. 219-228. - GANDOLFO, 2000a : Francesco Gandolfo, "Il Giubileo del 1300 e la Loggia delle benedizioni al Laterano ", dans Enzo Esposito éd., Dante e il Giubileo, (colloque, Rome, 1999), Florence, 2000, p. 201-214.

- GANDOLFO, 2000b : Francesco Gandolfo, "Il ritratto di committenza ", dans Maria Andaloro, Serena Romano éd., Arte e iconografia a Roma: da Costantino a Cola di Rienzo, Milan, 2000, p. 139-149. - GARDNER, 1969 : Julian Gardner, The Influence of Popes' and Cardinals' Patronage on the Introduction of the Gothic Style into Rome, 1254-1305, thèse, Courtauld Institute, Londres, 1969 (publication en cours).

- GARDNer, 1989a : Julian Gardner, "Patterns of Papal Patronage circa 1260 -circa $1300 "$ ", dans Christopher Ryan éd., The Religious Roles of the Papacy: Ideals and Realities, 1150-1300, Toronto, 1989, p. 439-456.

- GARDNER, 1989b : Julian Gardner, "Päpstliche Träume und Palastmalereien. Ein Essay über mittelalterliche Traumikonographie ", dans Agostino Paravicini Bagliani, Giorgio Stabile éd., Träume im Mittelalter: Ikonologische Studien, Stuttgart/Zurich, 1989, p. 113-124.

- GARdNer, 1990 : Julian Gardner, "The French Connection: Thoughts about French Patrons in Italian Art, c. 1250-1300", dans Charles Rosenberg éd., Art and Politics in Late Medieval and Early Renaissance Italy, 1250-1500, Londres, 1990, p. 81-102.

- GARDNER, 1992 : Julian Gardner, The Tomb and the Tiara: Curial Tomb Sculpture in Rome and Avignon in the Later Middle Ages, Oxford/New York, 1992.

- GARDNER, 1995 : Julian Gardner éd., Sancta Sanctorum, Milan, 1995.

- GARDNER, 1999a : Julian Gardner, "The Artistic Patronage of Pope Nicholas IV ", dans Anna Rosa Calderoni Masetti éd., Oreficerie e smalti in Europa fra XIII e XV secolo, (colloque, Pise, 1996), Pise, 1999, p. 1-8.

- GARDNER, 1999b : Julian Gardner, "The Artistic Patronage of Boniface VIII. The Perugian Inventory of the Papal Treasure of 1311 ", dans 'Roma caput mundi': Apogeo e declino di Roma medievale, Rome, 1999, p. 69-87.

- GARDNER, 2003a : Julian Gardner,

"Innocent III and His Influence on 
Roman Art of the Thirteenth Century ", dans Andrea Sommerlechner éd., Innocenzo III: Urbs et Orbis, II, (colloque, Rome, 1998), Rome, 2003, p. 1245-1260.

- GARDNER, 2003b : Julian Gardner, "Likeness and/or Representation in English and French Royal Portraits c. 1250c. 1300 ", dans Martin Büchsel, Peter Schmidt éd., Das Porträt vor der Erfindung des Porträts, Mayence, 2003, p. 141-151.

- GARDNER, 2004 : Julian Gardner, "Opus Anglicanum, Goldsmithswork, Manuscript Illumination and Ivories in the Rome of Boniface VIII ", dans Le culture di Bonifacio VIII, (colloque, Bologne, 2004), Rome, 2004, p. 163-179.

- GARDNer, 2007 : Julian Gardner, "Stone Saints. Commemoration and Likeness in Thirteenth-Century Italy, France, and Spain ", dans Gesta, 46, 2007, p. 121-134.

- GAUdemet, 1985 : Jean Gaudemet, "Ubi papa, ibi Roma?", dans Pierangelo Catalano, Paolo Siniscalco éd., Roma fuori di Roma: istituzioni e immagini, (colloque, Rome, 1985), Rome, 1985, p. 69-80. - Gauthier, 1968 : Marie-Madeleine Gauthier, "La clôture émaillée de la Confession de Saint Pierre au Vatican lors du concile du Latran IV, 1215", dans André Grabar et al., Synthronon : art et archéologie de la fin de l'Antiquité et du Moyen Âge, Paris, 1968, p. 237-248.

- HAGeNEDer, 2000 : Othmar Hageneder, Il sole e la luna: papato, impero e regni nella teoria e nella prassi dei secoli XII e XIII, Maria Pia Alberzoni éd., Milan, 2000.

- Herklotz, 1985 : Ingo Herklotz, 'Sepulcra' e 'monumenta' del Medioevo: studi sull'arte sepolcrale in Italia, Rome, 1985.

- HeRKLOTZ, 2000 : Ingo Herklotz, Gli eredi di Costantino: il papato, il Laterano e la propaganda visiva nel XII secolo, Rome, 2000.

- Herklotz, 2002 : Ingo Herklotz, "Bildpropaganda und monumentale Selbstdarstellungen des Papsttums ", dans Ernst-Dieter Hehl, Ingrid Heike Ringel, Hubertus Seibert éd., Das Papsttum in der Welt des 12. Jahrhunderts, Stuttgart, 2002, p. 273-291.

- IACOBIni, 1991 : Antonio Iacobini, "La pittura e le arti suntuarie da Innocenzo III a Innocenzo IV (1 198-1254) ", dans Angiola Maria Romanini éd., Roma nel Duecento: l'arte nella città dei papi da Innocenzo III a Bonifacio VIII, Turin, 1991, p. 237-319.

- IACOBINI, 1997 : Antonio Iacobini,

"Est haec sacra principis aedes: la basilica vaticana da Innocenzo III a Gregorio IX ", dans Quaderni dell'Istituto di storia dell'architettura, 25-30, 1995-1997, p. 91-100.

- KANTOROWICZ, 1957 : Ernst H. Kantorowicz, The King's Two Bodies: A Study in Mediaeval Political Theology, Princeton, 1957 [éd. fr. : Les Deux Corps du roi : essai sur la théologie politique au Moyen Âge, Paris, 1989].

- KANTOROWiCZ, 1961 : Ernst H. Kantorowicz, "The Sovereignty of the Artist. A Note on Legal Maxims and Renaissance Theories of Art ", dans 'De artibus opuscula XL': essays in Honor of Erwin Panofsky, New York, 1961, p. 267-279.

- KRAUTHEIMER, 2001 : Richard Krautheimer, Rome : portrait d'une ville, 312 1308, Paris, 2001.

- LADNER, 1941-1984 : Gerhard B. Ladner, Die Papstbildnisse des Altertums und des Mittelalters, 3 vol., Cité du Vatican, 1941-1984. - LADNER, 1983: Gerhart B. Ladner, " The Concepts of Ecclesia and Christianitas and their Relation to the Idea of Papal Plenitudo potestatis from Gregory VII to Boniface VIII ", dans Images and Ideas in the Middle Ages. Selected Studies in History and Art, II, Rome, 1983, p. 487-515.

- LAHARIE, 2008 : Muriel Laharie, Le Journal singulier d'Opicinus de Canistris (1337-vers 1341): Vaticanus latinus 6435, Cité du Vatican, 2008.

- LANZ, 1983 : Hanspeter Lanz, Die romanischen Wandmalereien von San Silvestro in Tivoli: Ein römisches Apsisprogramm der Zeit Innocenz III., Berne/ Francfort-sur-le-Main, 1983.

- Le Pogam, 2004 : Pierre-Yves Le Pogam, Les Maîtres d'auvre au service de la papauté dans la seconde moitié du XIII siècle, Rome, 2004.

- Le Pogam, 2005 : Pierre-Yves Le Pogam, De la Cité de Dieu au Palais du Pape : les résidences pontificales de la seconde moitié du XIII siècle, Rome, 2005.

- Le PogAM, 2008 : Pierre-Yves Le Pogam, " La lutte entre Boniface VIII et les Colonna par les armes symboliques ", dans Rivista di storia della Chiesa in Italia, 27, 2007, p. 47-66.

- Maccarrone, 1952 : Michele Maccarrone, 'Vicarius Christi': Storia del titolo papale, Rome, 1952.

- MACCARRONE et al., 1971 : Michele Maccarrone et al., La cattedra lignea di san Pietro in Vaticano: quattro studi, Cité du Vatican, 1971. - MACCARrone, 1975 : Michele Maccarrone, Nuove ricerche sulla cattedra lignea di san Pietro in Vaticano, Cité du Vatican, 1975.
- Maccarrone, 1983: Michele Maccarrone, "Ubi est papa, ibi est Roma", dans Aus Kirche und Reich: Studien zu Theologie, Politik und Recht im Mittelalter, Festschrift für Friedrich Kempf zu seinem fünfundsiebzigsten Geburtstag und fünfzigjährigen Doktorjubiläum, Sigmaringen, 1983, p. 371-82.

- MACCARRone, 1991 : Michele Maccarrone, Romana Ecclesia, Cathedra Petri, 2 vol., Rome, 1991.

- MAddalo, 1997 : Silvia Maddalo, « Roma miniata, Roma affrescata. Tracce di un mito fra Trecento e Quattrocento ", dans La storia dei Giubilei, I, 1300-1423, Florence, 1997, p. 118-133.

- MAdDALO, 1998-1999 : Silvia Maddalo, " Ancora sulla Loggia di Bonifacio VIII al Laterano. Una proposta di ricostruzione e un'ipotesi attributiva ", dans ArMed, II, 12-13, 1998-1999, p. 211-230.

- MAddalo, 2000 : Silvia Maddalo, « Da Magister Nicolaus al Maestro del codice di San Giorgio: linee di sviluppo del libro miniato a Roma nella seconda metà del Duecento ", dans Bonifacio VIII e il suo tempo: anno 1300 il primo Giubileo, Marina Righetti Tosti-Croce éd., (cat. expo., Rome, Palazzo di Venezia, 2000), Milan, 2000, p. 99-102.

- Melloni, 1990 : Alberto Melloni, Innocenzo IV: la concezione e l'esperienza della cristianità come regimen unius personae, Gênes, 1990.

- Miethie, 1991 : Jürgen Miethke, "Politische Theorien im Mittelalter", dans Hans-Joachim Lieber éd., Politische Theorien von der Antike bis zur Gegenwart, Bonn, 1991. - Mitchell, 1980 : John Mitchell, «St. Silvester and Constantine at the Santi Quattro Coronati ", dans Angiola Maria Romanini éd., Federico II e l'arte del Duecento italiano, I, Galatina, 1980, p. 15-32. - Monciatti, 2005 : Alessio Monciatti, Il palazzo vaticano nel Medioevo, Florence, 2005.

- NARdella, 1997 : Cristina Nardella, Il fascino di Roma nel Medioevo: Le Meraviglie di Roma di maestro Gregorio, Rome, 1997.

- Olariu, 2009 : Dominic Olariu éd., Le Portrait individuel: réflexions autour d'une forme de représentation, XIII'$X V^{e}$ siècles, Berne, 2009.

- Otavsky, 1986 : Karel Otavsky, "La Rose d'or du musée de Cluny", dans Revue du Louvre et des musées de France, 36, 1986, p. 379-385.

- PACE, 2000 : Valentino Pace, Arte a Roma nel Medioevo: committenza, ideologia 
e cultura figurativa in monumenti e libri, Naples, 2000.

- PARAVICINI BAgliani, 1987 : Agostino Paravicini Bagliani, "Ruggero Bacone autore del De retardatione accidentium senectutis?", dans Studi medievali, 3/28, 1987, p. 707-728.

- PARAVicini BAgliani, 1991 : Agostino Paravicini Bagliani, Medicina e scienze della natura alla corte dei papi nel Duecento, Spolète, 1991.

- PARAVICINI BAgliani, 1996 : Agostino Paravicini Bagliani, Il trono di Pietro: l'universalità del papato da Innocenzo III a Bonifacio VIII, Rome, 1996.

- PARAVICINI BAgliani, 1997 : Agostino Paravicini Bagliani, Le Corps du pape, Paris, 1997.

- PARAVICINI BAgliani, 1998 : Agostino Paravicini Bagliani, Le Chiavi e la Tiara: immagini e simboli del papato medievale, Rome, 1998.

- PARAVICINI BAgliani, 2003a : Agostino Paravicini Bagliani, " Ruggero Bacone e l'alchimia di lunga vita. Riflessioni sui testi ", dans Chiara Crisciani, Agostino Paravicini Bagliani éd., Alchimia e medicina nel Medioevo, Florence, 2003, p. 33-54.

- PARAVICINI BAgliani, 2003b : Agostino Paravicini Bagliani, Boniface VIII : un pape hérétique?, Paris, 2003.

- PARAVICINI BAgliani, 2005 : Agostino Paravicini Bagliani, "Bonifacio VIII, la Loggia di giustizia al Laterano e i processi generali di scomunica ", dans Rivista di storia della Chiesa in Italia, 59, 2005 , p. 377-428 [éd. augm. dans PARAVICINI BAGLIANI, 2009a, p. 153-214].

- PARAVICINI BAgliani, 2007 : Agostino Paravicini Bagliani, « Les portraits de Boniface VIII. Une tentative de synthèse ", dans PARAVICINI BAGLIANI, SPIESER, WIRTH, 2007, p. 117-139 [éd. augm., dans PARAVICINI BAGLIANI, 2009a, p. 116-136].

- PARAVicini Bagliani, 2009a : Agostino Paravicini Bagliani, Il potere del papa: corporeità, autorappresentazione, simboli, Florence, 2009.

- Paravicini Bagliani, 2009b : Agostino Paravicini Bagliani, "Il papato medievale e il concetto di Europa ", dans PARAVICINI BAGLIANI, 2009a, p. 293-314.

- PARAVICINI BAgLIANI, 2009c : Agostino PARAVICINI BAgLIANI, "Autour de la Rose d'or du comte de Neuchâtel au Musée de Cluny ", dans PARAvicinI BAGLIANI, 2009a, p. 347-54.

- PARAVICINI BAgLiani, 2009d: Agostino Paravicini Bagliani, " Riflessioni intorno alla paternità baconiana del Liber sex scientiarum", dans Chiara Crisciani,
Luciana Repici, Pietro B. Rossi éd., 'Vita longa': vecchiaia e durata della vita nella tradizione medica e aristotelica antica e medievale, Florence, 2009, p. 169-180.

- PARAVICINI BAgliani, 2010a : Agostino Paravicini Bagliani, "I baci liturgici del papa nel Medioevo. Prime ricerche ", dans Come l'orco della fiaba: studi per Franco Cardini, Florence, 2010, p. 533-544. - PARAVICINI BAgliani, 2010b : Agostino Paravicini Bagliani, Il papato nel secolo XIII: cent'anni di bibliografia, Florence, 2010.

- PARAVICINi BAgliani, SPIESER, WIRTH, 2007 : Agostino Paravicini Bagliani, Jean-Michel Spieser, Jean Wirth, Le Portrait : la représentation de l'individu, Florence, 2007.

- PARAVICINI BAGLIANI, WILLIAMS, 1987 : Agostino Paravicini Bagliani, Steven J. Williams, "Addendum ", dans Studi medievali, 3/28, 1987, p. 727-728.

- RASH, 1987 : Nancy Rash, “ Boniface VIII and Honorific Portraiture. Observations on the Half-Length Image in the Vatican ", dans Gesta, 26/1, 1987, p. 47-58.

- RÉfICE, 1990 : Paola Réfice, « Le Chiavi del regno: analisi documentarie e iconografiche sul San Pietro bronzeo vaticano ", dans Arte medievale, 2/4, 1990, p. 59-64.

- Romanini, 1988 : Angiola Maria Romanini, " Gli occhi di Isacco. Classicismo e curiosità scientifica tra Arnolfo di Cambio e Giotto ", dans Arte medievale, 2/2, 1988, p. 1-56.

- Romanini, 1990 : Angiola Maria Romanini, "Ipotesi ricostruttive per i monumenti sepolcrali di Arnolfo di Cambio ", dans Jörg Garms, Angiola Maria Romanini éd., Skulptur und Grabmal des Spätmittelalters in Rom und Italien, (colloque, Rome, 1985), Vienne, 1990, p. 107-128.

- Romanini, 1994 : Angiola Maria Romanini, "Une statue romaine dans la Vierge De Braye", dans Revue de l'art, 105, 1994, p. 9-18.

- Romanini, 1995a : Angiola Maria Romanini, "Arnolfo di Cambio nella basilica di San Pietro ", dans Quaderni dell'Istituto di storia dell'achitettura, 25-30, 1995-1997, p. 45-62.

- RoMANini, 1995b : Angiola Maria Romanini, "Gli occhi dipinti degli accoliti De Braye ", dans Napoli, l'Europa: ricerche di storia dell'arte in onore di Ferdinando Bologna, Catanzaro, 1995, p. 35-40.

- Romanini, 2001 : Angiola Maria Romanini, "L'attribuzione della statua bronzea di san Pietro al Vaticano ", dans La figura di san Pietro nelle fonti del Medioevo, (colloque, Viterbe/Rome, 2000), Louvain-la-Neuve, 2001, p. 549-568.

- RomAno, 1992 : Serena Romano, Eclissi di Roma: pittura murale a Roma e nel Lazio da Bonifacio VIII a Martino V (12951431), Rome, 1992.

- Romano, 1995 : Serena Romano, "Il Sancta Sanctorum: gli affreschi ", dans GARDNER, 1995, p. 38-125.

- Romano, 2000 : Serena Romano, "L'Acheropita lateranense: storia e funzione ", dans Il Volto di Cristo, (cat. expo., Rome, Palazzo delle esposizioni, 20002001), Milan, 2000, p. 39-41.

- ROMANO, 2000-2001 : Serena Romano, "Cristo, Niccolò III e l'antico ", dans Römisches Jahrbuch, 34, 2001-2002, p. 43-67. - Romano, 2001 : Serena Romano, La basilica di San Francesco ad Assisi: pittori, botteghe, strategie narrative, Rome, 2001.

- ROMANo, 2002 : Serena Romano, " L'icône Acheropoietes du Latran. Fonction d'une image absente ", dans Nicolas Bock, Peter Kurmann, Serena Romano éd., Art, cérémonial et liturgie au Moyen Âge, (colloque, Lausanne/Fribourg, 2000), Rome, 2002, p. 301-319.

- Romano, 2006 : Serena Romano, "Visione e visibilità nella Roma papale: Niccolò III e Bonifacio VIII ", dans Bonifacio VIII: ideologia e azione politica, (Bonifaciana, 2), (colloque, Rome, 2004), Rome, 2006, p. 61-76.

- Romano, 2008: Serena Romano, La O di Giotto, Milan, 2008.

- SCHIMMELPFENNIG, 1973 : Bernhard Schimmelpfennig, Die Zeremonienbücher der römischen Kurie im Mittelalter, (Bibliothek des Deutschen Historischen Instituts in Rom, 40), Tübingen, 1973.

- SCHIMMELPFENNig, 1990 : Bernard Schimmelpfennig, "Papal Coronations in Avignon ", dans János M. Bak éd., Coronations: Medieval and Early Modern Monarchic Ritual, Berkeley/Los Angeles/ Oxford, 1990, p. 179-196.

- SCHIMMELPFENNIG, 2005a : Bernard Schimmelpfennig, Papsttum und Heilige: Kirchenrecht und Zeremoniell, Ausgewählte Aufsätze, Georg Kreuzer, Stefan Weiss éd., Augsbourg, 2005.

- SCHIMMELPFENNig, 2005b : Bernard Schimmelpfennig, " Die Krönung des Papstes im Mittelalter dargestellt am Beispiel der Krönung Pius' II. (3.9.1458) ", dans SCHIMMELPFENNIG, 2005a, p. 68-132.

- SCHMidT, 1986 : Tilmann Schmidt, " Papst Bonifaz VIII. und die Idolatrie", 
dans Quellen und Forschungen aus italienischen Archiven und Bibliotheken, 66, 1986, p. 75-107.

- SCHRAMM, 1954-1956 : Percy Ernst Schramm, Herrschaftszeichen und Staatssymbolik: Beiträge zu ihrer Geschichte vom dritten bis zum sechzehnten Jahrhundert, 3 vol., Stuttgart, 1954-1956.

- SChramm, 1968-1971 : Percy Ernst Schramm, Kaiser, Könige und Päpste: Gesammelte Aufsätze zur Geschichte des Mittelalters, 4 vol., Stuttgart, 1968 1971.

- Silanos, 2010 : Pietro Silanos, Gerardo Bianchi da Parma: la biografia di un cardinale-legato duecentesco, Rome, 2010.

- TOMEI, 1990 : Alessandro Tomei, Iacobus Torriti pictor: una vicenda figurativa del tardo Duecento romano, Rome, 1990.

- Tomei, 1991 : Alessandro Tomei, "La pittura e le arti suntuarie da Alessandro IV a Bonifacio VIII (12541303) ", dans Angiola Maria Romanini éd., Roma nel Duecento: l'arte nella città dei papi da Innocenzo III a Bonifacio VIII, Turin, 1991, p. 321-403.

- Tomer, 1995 : Alessandro Tomei, "Un modello di committenza papale: Niccolò III e Roma ", dans GARDNER, 1995, p. 192-201.
- Tomei, 2000 : Alessandro Tomei, "Bonifacio VIII e il Giubileo del 1300: la Roma di Cavallini e di Giotto ", dans Bonifacio VIII e il suo tempo: anno 1300 il primo Giubileo, Marina Righetti Tosti-Croce éd., (cat. expo., Rome, Palazzo di Venezia, 2000), Milan, 2000, p. 93-98.

- Travaini, 2004 : Lucia Travaini, « La numismatica e le monete all'epoca di Bonifacio VIII ", dans Le culture di Bonifacio VIII, (colloque, Bologne, 2004), Rome, 2004, p. 195-214.

- VAuchez, 2002 : André Vauchez, "Les songes d'Innocent III ", dans Studi sulle società e le culture del Medioevo per Girolamo Arnaldi, II, Rome, 2002, p. 695-706. - Voci, 1992 : Anna Maria Voci, Nord o Sud? Note per la storia del medioevale Palatium apostolicum apud Sanctum Petrum e delle sue cappelle, Cité du Vatican, 1992.

- Weddigen, BlaAuw, Kempers, 2003 : Tristan Weddigen, Sible de Blaauw, Bram Kempers éd., Functions and Decorations: Art and Ritual at the Vatican Palace in the Middle Ages and the Renaissance, Cité du Vatican/Turnhout, 2003.

- Williams, 2003 : Steven J. Williams, The Secret of Secrets: The Scholarly Career of a Pseudo-Aristotelian Text in the Latin Middle Ages, Ann Arbor, 2003.

- Wolf, 1998: Gerhard Wolf, " From Mandylion to Veronica. Picturing the 'Disembodied' Face and Disseminating the True Image of Christ in the West ", dans The Holy Face and the Paradox of Representation, Bologne, 1998, p. 153-179.

- Wolf, 2000 : Gerhard Wolf, «La Veronica tra i santi Pietro e Paolo come timbro giubilare ", dans Mori Edoardo, Mori Elisabetta éd., Un documento cortonese sul Giubileo del '300: La lettera di Silvestro scriptor pontificio (carta 6 del codice 101), Cortone, 2000, p. 19-24.

- WOLF, 2007 : Gerhard Wolf, « Il corpo del papa e il volto di Cristo: un affresco di Urbano V in San Francesco a Terni ", dans Iconografica: Rivista di iconografia medievale e moderna, 6, 2007, p. 109-114.

\section{Mots-clés}

autoreprésentation, anthropologie culturelle, arts visuels, iconographie, symbolique du pouvoir 